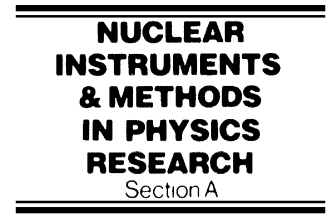

www.elsevier.nl/locate/nima

\title{
Hadronic shower development in Iron-Scintillator Tile Calorimetry
}

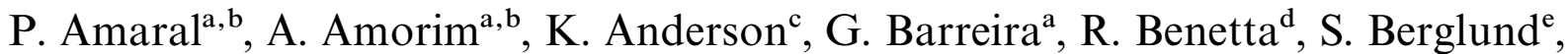

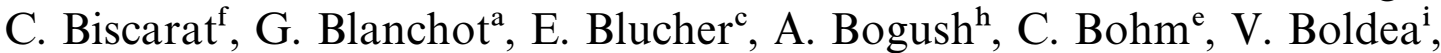
O. Borisov ${ }^{\mathrm{j}}$, M. Bosman ${ }^{\mathrm{g}}$, C. Bromberg ${ }^{\mathrm{k}}$, J. Budagov ${ }^{\mathrm{j}}$, S. Burdin ${ }^{1}$, L. Caloba ${ }^{\mathrm{m}}$, J. Carvalhon, P. Casadog, M.V. Castillo ${ }^{\mathrm{v}}$, M. Cavalli-Sforza ${ }^{\mathrm{g}}$, V. Cavasinni', R. Chadelas ${ }^{\mathrm{f}}$, I. Chirikov-Zorin ${ }^{\mathrm{j}}$, G. Chlachidze ${ }^{\mathrm{j}}, \mathrm{M}$. Cobal ${ }^{\mathrm{d}}$, F. Cogswell ${ }^{\mathrm{p}}$, F. Colaço ${ }^{q}$, S. Cologna ${ }^{1}$, S. Constantinescu ${ }^{\mathrm{i}}$, D. Costanzo ${ }^{1}$, M. Crouau ${ }^{\mathrm{f}}$, F. Daudon ${ }^{\mathrm{f}}$,

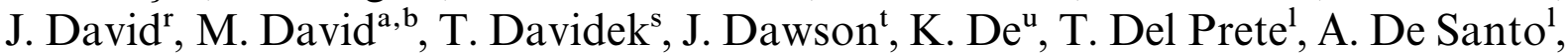
B. Di Girolamo ${ }^{1}$, S. Dita ${ }^{\mathrm{i}}$, J. Dolejsis ${ }^{\mathrm{s}}$, Z. Dolezal ${ }^{\mathrm{s}}$, R. Downing ${ }^{\mathrm{p}}$, I. Efthymiopoulos ${ }^{\mathrm{g}}$, M. Engström ${ }^{\mathrm{e}}$, D. Errede ${ }^{\mathfrak{p}}$, S. Errede ${ }^{\mathfrak{p}}$, H. Evans ${ }^{\mathrm{e}}$, A. Fenyuk ${ }^{\mathrm{r}}$, A. Ferrer ${ }^{\mathrm{v}}$, V. Flaminio, E. Gallas ${ }^{\mathrm{u}}$, M. Gaspar ${ }^{\mathrm{m}}$, I. Gil ${ }^{\mathrm{v}}$, O. Gildemeister ${ }^{\mathrm{d}}$, V. Glagolev ${ }^{\mathrm{j}}$, A. Gomes ${ }^{\mathrm{a}, \mathrm{b}}$, V. Gonzalez ${ }^{\mathrm{v}}$, S. González De La Hoz ${ }^{\mathrm{v}}$, V. Grabskiw ${ }^{\mathrm{w}}$, E. Grauges P. Grenier ${ }^{f}$, H. Hakopian ${ }^{w}$, M. Haney ${ }^{p}$, M. Hansen ${ }^{\text {, }}$ S. Hellman ${ }^{\mathrm{e}}$, A. Henriques ${ }^{\mathrm{a}}$, C. Hebrard ${ }^{\mathrm{f}}$, E. Higon , S. Holmgren ${ }^{\mathrm{e}}$, J. Huston ${ }^{\mathrm{k}}$, Yu. Ivanyushenkov ${ }^{\mathrm{g}}$, K. Jon-And ${ }^{\mathrm{e}}$, A. Juste ${ }^{\mathrm{g}}$, S. Kakurin ${ }^{\mathrm{j}}$, G. Karapetian ${ }^{\mathrm{d}}$, A. Karyukhin ${ }^{\mathrm{r}}$, S. Kopikov', V. Kukhtin ${ }^{j}$, Y. Kulchitsky ${ }^{\text {d,j }}$, W. Kurzbauer ${ }^{\mathrm{d}}$, M. Kuzmin ${ }^{\text {h }}$, S. Lamil, V. Lapin ${ }^{\mathrm{r}}$, C. Lazzeroni ${ }^{1}$, A. Lebedev ${ }^{j}$, R. Leitner ${ }^{\mathrm{s}}$, J. Li $^{\mathrm{u}}$, Yu. Lomakin ${ }^{\mathrm{j}}$, O. Lomakina ${ }^{\mathrm{j}}$, M. Lokajicek ${ }^{\mathrm{x}}$, J.M. Lopez Amengual ${ }^{\mathrm{v}}$, A. Maio ${ }^{\mathrm{a}, \mathrm{b}}$, S. Malyukov ${ }^{\mathrm{j}}$, F. Marroquin ${ }^{\mathrm{m}}$, J.P. Martins ${ }^{\mathrm{a}, \mathrm{b}}$, E. Mazzoni ${ }^{1}$, F. Merritt ${ }^{\mathrm{c}}$, R. Miller ${ }^{\mathrm{k}}$, I. Minashvili ${ }^{\mathrm{j}}$, Ll. Miralles ${ }^{\mathrm{g}}$,

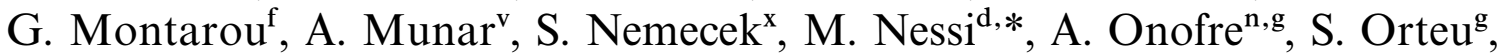
I.C. Park ${ }^{\mathrm{g}}$, D. Pallin ${ }^{\mathrm{f}}$, D. Pantea ${ }^{\mathrm{i}, \mathrm{j}}$, R. Paoletti ${ }^{1}$, J. Patriarca ${ }^{\mathrm{a}}$, A. Pereira ${ }^{\mathrm{m}}$, J.A. Perlas ${ }^{\mathrm{g}}$, P. Petit ${ }^{\mathrm{g}}$, J. Pilcher ${ }^{\mathrm{c}}$, J. Pinhão ${ }^{\mathrm{n}}$, L. Poggiolid ${ }^{\mathrm{d}}$, L. Price ${ }^{\mathrm{t}}$, J. Proudfoot ${ }^{\mathrm{t}}$, O. Pukhov ${ }^{\mathrm{j}}$, G. Reinmuth ${ }^{\mathrm{f}}$, G. Renzoni ${ }^{\mathrm{l}}$, R. Richards ${ }^{\mathrm{k}}$, C. Roda ${ }^{1}$, J.B. Romance, V. Romanov ${ }^{\mathrm{j}}$, B. Ronceux ${ }^{\mathrm{g}}$, P. Rosnet ${ }^{\mathrm{f}}$, V. Rumyantsev ${ }^{\mathrm{b}, \mathrm{j}}$, N. Russakovich ${ }^{\mathrm{j}}$, E. Sanchis ${ }^{v}$, H. Sanders ${ }^{c}$, C. Santonif ${ }^{f}$ J. Santos ${ }^{a}$, L. Sawyer ${ }^{\mathrm{u}}$, L.-P. Says ${ }^{\mathrm{f}}$, J.M. Seixas ${ }^{\mathrm{m}}$, B. Selldèn ${ }^{\mathrm{e}}$, A. Semenov ${ }^{\mathrm{j}}$, A. Shchelchkov ${ }^{\mathrm{j}}$, M. Shochet ${ }^{\mathrm{f}}$, V. Simaitis ${ }^{\mathrm{p}}$, A. Sissakian ${ }^{j}$, A. Solodkov ${ }^{r}$, O. Solovianov ${ }^{r}$, P. Sonderegger ${ }^{\mathrm{d}}$, M. Sosebee ${ }^{\mathrm{u}}$,

*Corresponding author. Tel.: + 41-22-767-2470; fax: + 41-22-767-6111.

E-mail address: marzio.nessi@cern.ch (M. Nessi). 
K. Soustruznik ${ }^{\mathrm{s}}$, F. Spanó ${ }^{1}$, R. Stanek ${ }^{\mathrm{t}}$, E. Starchenko ${ }^{\mathrm{r}}$, R. Stephens ${ }^{\mathrm{u}}$, M. Suk $^{\mathrm{s}}$,

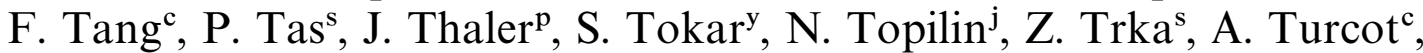

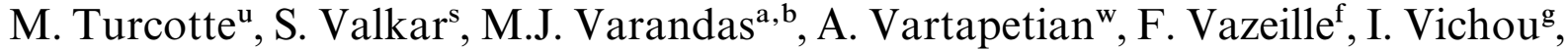
V. Vinogradov ${ }^{j}$, S. Vorozhtsov ${ }^{j}$, D. Wagner ${ }^{\mathrm{e}}$, A. White ${ }^{\mathrm{u}}$, H. Wolters ${ }^{\mathrm{q}}$, N. Yamdagni ${ }^{\mathrm{e}}$, G. Yarygin ${ }^{\mathrm{j}}$, C. Yosef ${ }^{\mathrm{k}}$, A. Zaitsev ${ }^{\mathrm{r}}$, M. Zdrazil ${ }^{\mathrm{s}}$, J. Zuñiga ${ }^{\mathrm{v}}$

${ }^{a}$ LIP Lisbon, Portugal

${ }^{\mathrm{b}}$ FCUL University of Lisbon, Portugal

${ }^{\mathrm{c}}$ University of Chicago, Chicago, IL, USA

${ }^{\mathrm{d}}$ CERN, Geneva, Switzerland

eStockholm University, Stockholm, Sweden

${ }^{\mathrm{f}}$ LPC Clermont-Ferrand, Université Blaise Pascal/CNRS-IN2P3, Clermont-Ferrand, France

${ }^{\mathrm{g}}$ Institut de Fisica d'Altes Energies, Universitat Autònoma de Barcelona, Barcelona, Spain

${ }^{\mathrm{h}}$ Institute of Physics, National Academy of Science, Minsk, Byelorussia

${ }^{\mathrm{i} N a t i o n a l ~ I n s t i t u t e ~ f o r ~ P h y s i c s ~ a n d ~ N u c l e a r ~ E n g i n e e r i n g, ~ B u c h a r e s t, ~ R o m a n i a ~}$

jJINR, Dubna, Russia

${ }^{\mathrm{k}}$ Michigan State University, East Lansing, MI, USA

${ }^{1}$ Pisa University and INFN, Pisa, Italy

${ }^{\mathrm{m}}$ COPPE/EE/UFRJ, Rio de Janeiro, Brazil

${ }^{\mathrm{n}}$ LIP and FCTUC Univ. of Coimbra, Portugal

${ }^{\mathrm{p}}$ University of Illinois, Urbana-Champaign, IL, USA

${ }^{\mathrm{q}}$ University Católica Figueira da Foz, Portugal

${ }^{\mathrm{r}}$ Institute for High Energy Physics, Protvino, Russia

${ }^{\mathrm{s}}$ Charles University, Prague, Czech Republic

'Argonne National Laboratory, Argonne, IL, USA

${ }^{\mathrm{u}}$ University of Texas at Arlington, Arlington, TX, USA

vIFIC, Centro Mixto Universidad de Valencia-CSIC, E46100 Burjassot, Valencia, Spain

${ }^{\mathrm{w}}$ Yerevan Physics Institute, Yerevan, Armenia

${ }^{\mathrm{x}}$ Academy of Science, Prague, Czech Republic

${ }^{y}$ Comenius University, Bratislava, Slovak Republic

Received 30 March 1999; received in revised form 13 September 1999; accepted 20 September 1999

\begin{abstract}
The lateral and longitudinal profiles of hadronic showers detected by a prototype of the ATLAS Iron-Scintillator Tile Hadron Calorimeter have been investigated. This calorimeter presents a unique longitudinal configuration of scintillator tiles. Using a fine-grained pion beam scan at $100 \mathrm{GeV}$, a detailed picture of transverse shower behaviour is obtained. The underlying radial energy densities for the four depth segments and for the entire calorimeter have been reconstructed. A three-dimensional hadronic shower parametrisation has been developed. The results presented here are useful for understanding the performance of iron-scintillator calorimeters, for developing fast simulations of hadronic showers, for many calorimetry problems requiring the integration of a shower energy deposition in a volume and for future calorimeters design. (C) 2000 Elsevier Science B.V. All rights reserved.
\end{abstract}

Keywords: Calorimetry; Computer data analysis

\section{Introduction}

We report on an experimental study of hadronic shower profiles detected by the prototype of the
ATLAS Barrel Tile Hadron Calorimeter (Tile calorimeter) $[1,2]$. The innovative design of this calorimeter, using longitudinal segmentation of active and passive layers (see Fig. 1) which has detailed 
description in Section 2, provides an interesting system for the measurement of hadronic shower profiles. Specifically, we have studied the transverse development of hadronic showers using $100 \mathrm{GeV}$ pion beams and the longitudinal development of hadronic showers using 20-300 GeV pion beams.

The characteristics of shower development in hadron calorimeters have been presented in several papers. However, a complete quantitative description of the transverse and longitudinal properties of hadronic showers does not exist [3]. The transverse profiles are usually expressed as a function of the transverse coordinates, not the radius, and are integrated over the other coordinate [4]. The threedimensional parametrisation of hadronic showers described here could be a useful starting point for fast simulations, which can be faster than full simulations at the microscopic level by several orders of magnitude [5-7].

The paper is organised as follows. In Section 2, the calorimeter and the beam test setup are briefly described. In Section 3, the mathematical procedures for extracting the underlying radial energy density of hadronic showers are developed. The obtained results on the transverse and longitudinal profiles, the radial energy densities and the radial containment of hadronic shower are presented in Sections 4-7. Sections 8 and 9 investigate the three-dimensional parametrisation and electromagnetic fraction of hadronic shower. Finally, Section 10 contains a summary and the conclusions.

\section{The calorimeter}

The Tile Calorimeter is a sampling device made out of steel and scintillating tiles, as absorber and active material, respectively [8]. A conceptual design of this calorimeter geometry is shown in Fig. 1. The absorber structure is a laminate of steel plates of various dimensions stacked along $Z$. The basic geometrical element of the stack is what we call a period. It consists of a set of two master plates (large trapezoidal steel plates, $5 \mathrm{~mm}$ thick, spanning along the entire $X$ dimension) and one set of spacer plates (small trapezoidal steel plates, $4 \mathrm{~mm}$ thick, $10 \mathrm{~cm}$ wide along $X$ ). During construction, half-

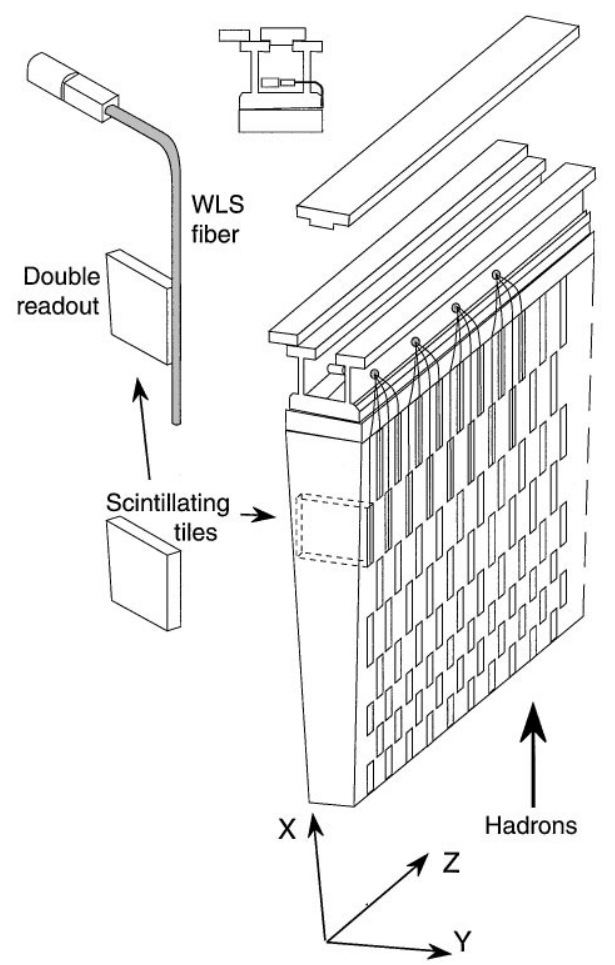

Fig. 1. Conceptual design of a Tile Calorimeter module.

period elements are pre-assembled starting from an individual master plate and the corresponding nine spacer plates. The relative position of the spacer plates in the two half periods is staggered in the $X$ direction, to provide pockets in the structure for the subsequent insertion of the scintillating tiles. Each stack, called module, spans $2 \pi / 64$ in the azimuthal angle ( $Y$ dimension), $100 \mathrm{~cm}$ in the $Z$ direction and $180 \mathrm{~cm}$ in the $X$ direction (about nine interaction lengths, $\lambda_{I}$, or about 80 effective radiation lengths, $X_{0}$ ). Each module has 57 repeated periods. The module front face, exposed to the beam particles, covers $100 \times 20 \mathrm{~cm}^{2}$. The scintillating tiles are made out of polystyrene material of thickness $3 \mathrm{~mm}$, doped with scintillator. The iron to scintillator ratio is $4.67: 1$ by volume. The calorimeter thickness along the beam direction at the incidence angle of $\Theta=10^{\circ}$ (the angle between the incident particle direction and the normal to the calorimeter front face) corresponds to $1.49 \mathrm{~m}$ of iron equivalent length [9]. 


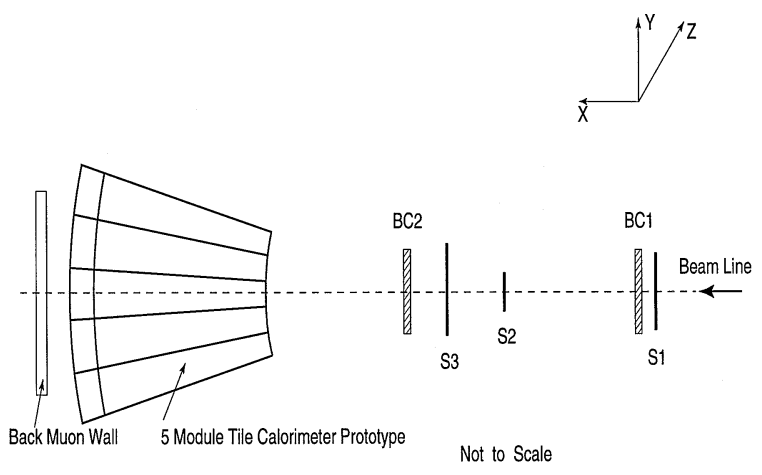

Fig. 2. Schematic layout of the experimental set-up (side view). $\mathrm{S} 1, \mathrm{~S} 2$ and $\mathrm{S} 3$ are beam trigger scintillators. $\mathrm{BC} 1$ and $\mathrm{BC} 2$ are $(\mathrm{Z}$, Y) proportional chambers.

Wavelength shifting fibres collect the scintillation light from the tiles at both of their open (azimuthal) edges and bring it to photo-multipliers (PMTs) at the periphery of the calorimeter (Fig. 1). Each PMT views a specific group of tiles through the corresponding bundle of fibres. The prototype Tile Calorimeter used for this study is composed of five modules stacked in the $Y$ direction, as shown in Fig. 2.

The modules are divided into five segments along $Z$ and they are also longitudinally segmented (along $X$ ) into four depth segments, as shown in Fig. 3. The readout cells have a lateral dimension of $200 \mathrm{~mm}$ along $Z$, and longitudinal dimensions of $300,400,500,600 \mathrm{~mm}$ for depth segments 1-4, corresponding to $1.5,2,2.5$ and $3 \lambda_{I}$ at $\Theta=0^{\circ}$ respectively. Along $Y$, the cell sizes vary between about 200 and $370 \mathrm{~mm}$ depending on the $X$ coordinate (Fig. 2). The energy release in 100 different cells was recorded for each event [8].

The calorimeter was placed on a scanning table that allowed movement in any direction. Upstream of the calorimeter, a trigger counter telescope (S1, S2, S3) was installed, defining a beam spot approximately $20 \mathrm{~mm}$ in diameter. Two delay-line wire chambers (BC1 and $\mathrm{BC} 2)$, each with $(Z, Y)$ readout, allowed the impact point of beam particles on the calorimeter face to be reconstructed to better than $\pm 1 \mathrm{~mm}$ [10]. Additional scintillators for the muon detection were placed behind and on the side of the calorimeter prototype to measure the longitudinal and lateral hadronic shower leakage [11]. The

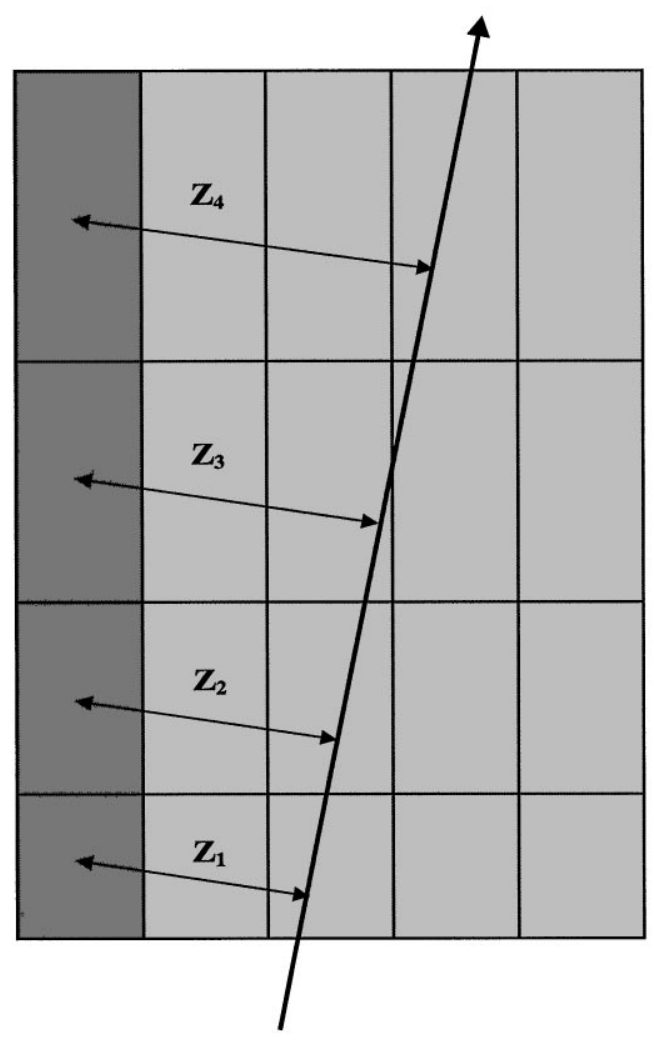

Fig. 3. Schematic layout (top view) of Tile Calorimeter prototype. $z_{1}-z_{4}$ are the distances between the centre of towers (for the four depth segments) and a point normal to a given beam particle track.

"Back Muon Wall" $\left(800 \times 800 \mathrm{~mm}^{2}\right)$ is shown in Fig. 2. The other scintillators were placed on the positive $Z$ side $\left(400 \times 1150 \mathrm{~mm}^{2}\right)$, and are not seen in Fig. 2.

The data used for the study of lateral profiles were collected in 1995 during a special $Z$-scan run at the CERN SPS test beam. The calorimeter was exposed to $100 \mathrm{GeV}$ negative pions at a $10^{\circ}$ angle with varying impact points in the $Z$-range from -360 to $+200 \mathrm{~mm}$. A total of $>300000$ events have been analysed; for the lateral profile study only events without lateral leakage were used. The uniformity of the calorimeter's response for this $Z$-scan is estimated to be $1 \%$ [12]. The data used for the study of longitudinal profiles were obtained using $20-300 \mathrm{GeV}$ negative pions at a $20^{\circ}$ angle and were taken during the same test beam run. 


\section{Extracting the underlying radial energy density}

In this investigation we use a coordinate system based on the incident particle direction. The impact point of the incident particle at the calorimeter front face defines the origin of the coordinate system. The incident particle direction forms the $x$-axis, while the $y$-axis is in the same direction as $Y$ defined in Section 2. The normal to the $x y$ surface defines the $z$-axis.

We measure the energy deposition in each calorimeter cell for every event. In the $i j k$-cell of the calorimeter with volume $V_{i j k}$ and cell center coordinates $\left(x_{c}, y_{c}, z_{c}\right)$, the energy deposition $E_{i j k}$ is

$E_{i j k}\left(x_{c}, y_{c}, z_{c}\right)=\int_{V_{i j k}} f(x, y, z) \mathrm{d} x \mathrm{~d} y \mathrm{~d} z$

where $f(x, y, z)$ is the three-dimensional hadronic shower energy density function. Due to the azimuthal symmetry of shower profiles, the density $f(x, y, z)$ is only a function of the radius $r=\sqrt{y^{2}+z^{2}}$ from the shower axis and the longitudinal coordinate $x$. Then

$E_{i j k}\left(x_{c}, y_{c}, z_{c}\right)=\int_{V_{i j k}} \Psi(x, r) r \mathrm{~d} r \mathrm{~d} \phi \mathrm{d} x$

where $\phi$ is the azimuthal angle and $\Psi(x, r)$ has the form of a joint probability density function (p.d.f) [13]. The joint p.d.f. can be further decomposed as a product of the marginal p.d.f., $\mathrm{d} E(x) / \mathrm{d} x$, and the conditional p.d.f., $\Phi(x, r)$,

$\Psi(x, r)=\frac{\mathrm{d} E(x)}{\mathrm{d} x} \Phi(x, r)$.

The longitudinal density $\mathrm{d} E / \mathrm{d} x$ is defined as

$\frac{\mathrm{d} E(x)}{\mathrm{d} x}=\int_{-\infty}^{\infty} \int_{-\infty}^{\infty} f(x, y, z) \mathrm{d} y \mathrm{~d} z$.

Finally, the radial density function $\Phi(r)$ for a given depth segment is

$\Phi(r)=E_{0} \Phi(x, r)$

where $E_{0}$ is the total shower energy deposition into the depth segment for fixed $x$.
There are several methods for extracting the radial density $\Phi(r)$ from the measured distributions of energy depositions. One method is to unfold $\Phi(r)$ using expression (2). This method was used in the analysis of the data from the lead-scintillating fibre calorimeter [14]. Several analytic forms of $\Phi(r)$ were tried, but the simplest that describes the energy deposition in cells was a combination of an exponential and a Gaussian:

$\Phi(r)=\frac{b_{1}}{r} \mathrm{e}^{-r / \mu_{1}}+\frac{b_{2}}{r} \mathrm{e}^{-\left(r / \mu_{2}\right)^{2}}$

where $b_{i}$ and $\mu_{i}$ are the free parameters.

Another method for extracting the radial density is to use the marginal density function

$f(z)=\int_{-\infty}^{\infty} \int_{x_{1}}^{x_{2}} f(x, y, z) \mathrm{d} x \mathrm{~d} y$.

which is related to the radial density $\Phi(r)$.

$f(z)=2 \int_{|z|}^{\infty} \frac{\Phi(r) r \mathrm{~d} r}{\sqrt{r^{2}-z^{2}}}$.

This method was used [15] for extracting the electron shower transverse profile from the GAMS2000 electromagnetic calorimeter data [16]. The above integral equation (8) can be reduced to an Abelian equation by replacing variables [17]. In Ref. [18], the following solution to Eq. (8) was obtained:

$\Phi(r)=-\frac{1}{\pi} \frac{\mathrm{d}}{d r^{2}} \int_{r^{2}}^{\infty} \frac{f(z) \mathrm{d} z^{2}}{\sqrt{z^{2}-r^{2}}}$.

For our study, we used the sum of three exponential functions to parameterise $f(z)$ as

$f(z)=\frac{E_{0}}{2 B} \sum_{i=1}^{3} a_{i} \mathrm{e}^{-|z| / \lambda_{i}}$

where $z$ is the transverse coordinate, $E_{0}, a_{i}, \lambda_{i}$ are free parameters, $B=\sum_{i=1}^{3} a_{i} \lambda_{i}, \sum_{i=1}^{3} a_{i}=1$ $\int_{-\infty}^{+\infty} f(z) \mathrm{d} z=E_{0}$. In this case, the radial density function, obtained by integration and differentiation of Eq. (9), is

$\Phi(r)=\frac{E_{0}}{2 \pi B} \sum_{i=1}^{3} \frac{a_{i}}{\lambda_{i}} K_{0}\left(\frac{r}{\lambda_{i}}\right)$ 
where $K_{0}$ is the modified Bessel function. This function goes to $\infty$ as $r \rightarrow 0$ and goes to zero as $r \rightarrow \infty$.

We define a column of five cells in a depth segment as a tower. Using the parametrisation shown in Eq. (10), we can show that the energy deposition in a tower, $E(z)=\int_{z-h / 2}^{z+h / 2} f(z) \mathrm{d} z$, can be written as

$E(z)=E_{0}-\frac{E_{0}}{B} \sum_{i=1}^{3} a_{i} \lambda_{i} \cosh \left(\frac{|z|}{\lambda_{i}}\right) \mathrm{e}^{-h / 2 \lambda_{i}}$ for $|z| \leqslant \frac{h}{2}$

$E(z)=\frac{E_{0}}{B} \sum_{i=1}^{3} a_{i} \lambda_{i} \sinh \left(\frac{h}{2 \lambda_{i}}\right) \mathrm{e}^{-|z| / \lambda_{i}} \quad$ for $|z| \leqslant \frac{h}{2}$

where $h$ is the size of the front face of the tower along the $z$-axis. Note that as $h \rightarrow 0$, we get $E(z) / h \rightarrow f(z)$. As $h \rightarrow \infty$, we find that $E(0) \rightarrow E_{0}$.

The full-width at the half-maximum (FWHM) of an energy deposition profile for small values of $(\mathrm{FWHM}-h) / h$ can be approximated by

$F W H M=h+\frac{2 E(h / 2)-E(0)}{\left(E_{0} / B\right) \sum_{i=1}^{3} a_{i} \sinh \left(h / 2 \lambda_{i}\right) \mathrm{e}^{-h / 2 \lambda_{i}}}$.

We will show below that this approximation agrees well with our data.

A cumulative function may be derived from the density function as

$F(z)=\int_{-\infty}^{z} f(z) \mathrm{d} z$

For our parametrisation in Eq. (10), the cumulative function becomes

$$
\begin{aligned}
& F(z)=\frac{E_{0}}{2 B} \sum_{i=1}^{3} a_{i}, \lambda_{i} \mathrm{e}^{z / \lambda_{i}} \quad \text { for } z \leqslant 0 \\
& F(z)=E_{0}-\frac{E_{0}}{2 B} \sum_{i=1}^{3} a_{i}, \lambda_{i} \mathrm{e}^{-z / \lambda_{i}} \quad \text { for } z>0
\end{aligned}
$$

where $z$ is the position of the edge of a tower along the $z$-axis. Note that the cumulative function does not depend on the cell size $h$. We can construct the cumulative function and deconvolute the density $f(z)$ from it for any size calorimeter cell. Note also that cumulative function is well behaved at the key points: $F(-\infty)=0, F(0)=E_{0} / 2$, and $F(\infty)=E_{0}$.

The radial containment of a shower as a function of $r$ is given by

$I(r)=\int_{0}^{r} \int_{0}^{2 \pi} \Phi(r) r \mathrm{~d} r \mathrm{~d} \phi=E_{0}-\frac{E_{0} r}{B} \sum_{i=1}^{3} a_{i} K_{1}\left(\frac{r}{\lambda_{i}}\right)$

where $K_{1}$ is the modified Bessel function. As $r \rightarrow \infty$, the function $r K_{1}(r)$ tends to zero and we get $I(\infty)=E_{0}$, as expected.

We use two methods to extract the radial density function $\Phi(r)$. One method is to unfold $\Phi(r)$ from Eq. (2). Another method is to use the expression (9) after we have obtained the marginal density function $f(z)$. There are three ways to extract $f(z)$ : by fitting the energy deposition $E(z)$, by fitting the cumulative function $F(z)$ and by directly extracting $f(z)$ by numerical differentiation of the cumulative function. The effectiveness of these various methods depends on the scope and quality of the experimental data.

\section{Transverse behaviour of hadronic showers}

Fig. 4 shows the energy depositions in towers for depth segments $1-4$ as a function of the $z$ coordinate of the centre of the tower. Fig. 5 shows the same for the entire calorimeter (the sum of the histograms presented in Fig. 4). Here the coordinate system is linked to the incident particle direction where $z=0$ is the coordinate of the beam impact points at the calorimeter front face. Fig. 3 schematically shows a top view of the experimental set-up and indicates the $z$ coordinate of some towers. The $z_{1}, z_{2}, z_{3}$ and $z_{4}$ are the distances between the centre of towers (for the four depth segments) and a point normal to a given beam particle track. The values of the $z$ coordinate of the tower centres are negative to the left of the beam, positive to the right of the beam. For any given event, we can define only five tower energy depositions for each depth segment and $z$ coordinates are 

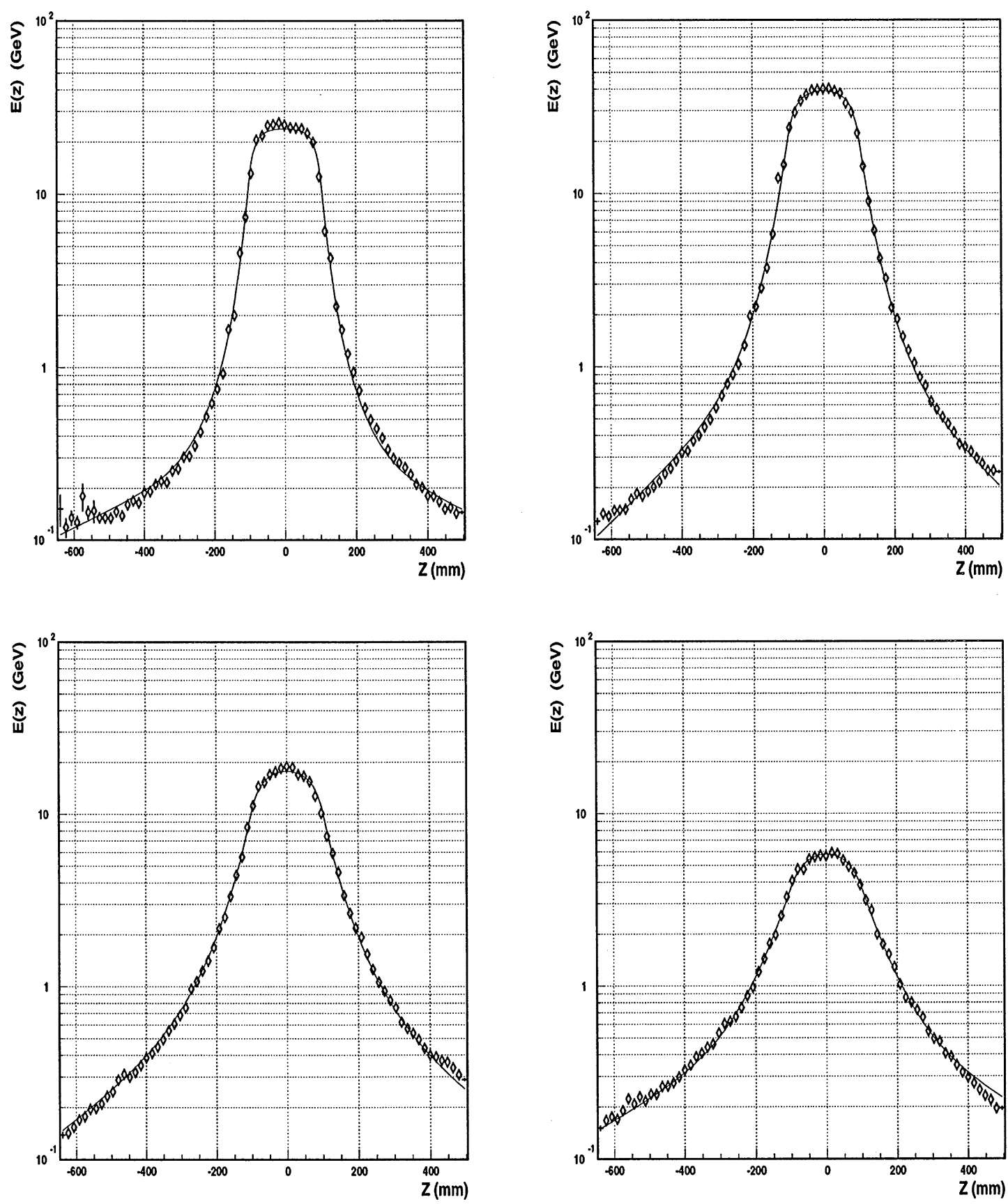

Fig. 4. Energy depositions of $100 \mathrm{GeV}$ pions in towers of depth segments $1-4$ as a function of the $z$ coordinate: top left is for depth segment 1 , top right is for depth segment 2 , bottom left is for depth segment 3 , bottom right is for depth segment 4 . Only statistical errors are shown. Curves are fits of Eqs. (12) and (13) to the data. 


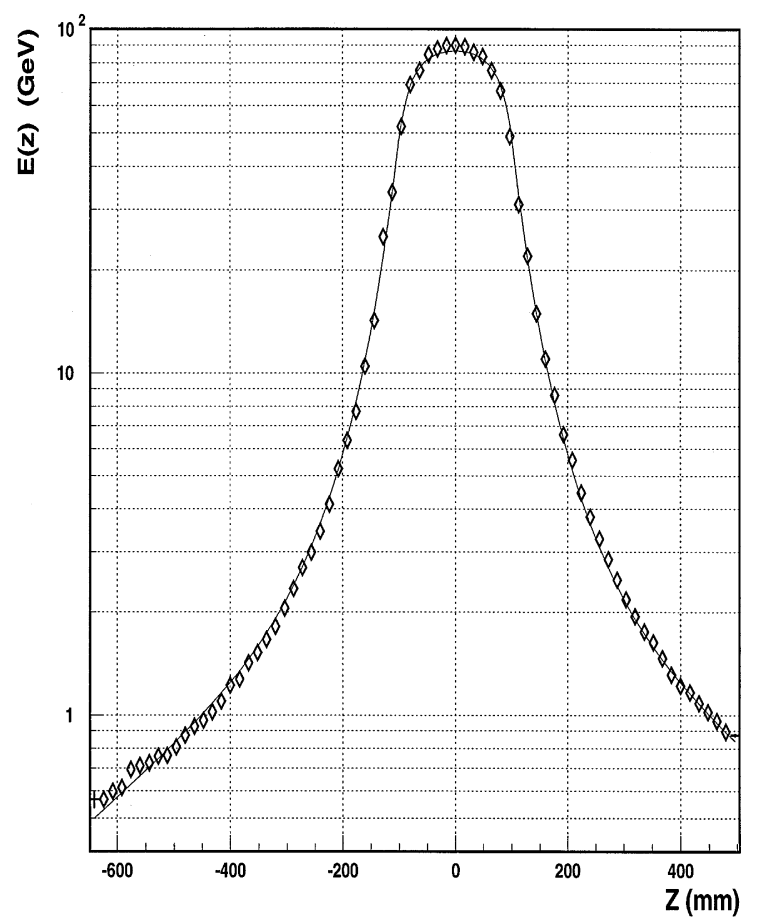

Fig. 5. Energy depositions in towers, summed over all calorimeter depth segments, as a function of the $z$ coordinate. Only statistical errors are shown. The curve is the result of the fit by formulas (12) and (13).

quantised with step size equal to the cell width (at $10^{\circ}$ in our case). However, with fine parallel displacements of the beam between -360 and $200 \mathrm{~mm}$ ( $Z$-scan) we expand the tower coordinate range from -760 to $600 \mathrm{~mm}$. To avoid edge effects, we present tower energy depositions in the range from -650 to $500 \mathrm{~mm}$. For our entire data sample, we have more than $10^{6}$ tower energy measurements for each depth segment. Figs. 4 and 5 are profile histograms and give the energy deposited in any tower for all analysed events in bins of the $z$ coordinate. Note that the total tower height (about $1.0 \mathrm{~m}$ at the front face, and about $1.8 \mathrm{~m}$ at the back) is sufficient for shower measurements without significant leakage in the vertical direction.

As mentioned earlier, events with significant lateral leakage (identified by a clear minimum-ionising signal in the lateral muon wall) were discarded. The resulting left-right asymmetries in the distributions of Figs. 4 and 5 are very small.
As will be shown later (Section 6), the $99 \%$ containment radius is less than $500 \mathrm{~mm}$.

The fine-grained $z$-scan provided many different beam impact locations within the calorimeter. Due to this, we obtained a detailed picture of the transverse shower behaviour in the calorimeter. The tower energy depositions shown in Figs. 4 and 5 span a range of about three orders of magnitude. The plateau for $|z|<100 \mathrm{~mm}(h / 2)$ and the fall-off at large $|z|$ are apparent. Similar behaviour of the transverse profiles was observed in other calorimeters as well $[14,19]$.

We used the distributions in Figs. 4 and 5 to extract the underlying marginal densities function for four depth segments of the calorimeter and for the entire calorimeter. The solid curves in these figures are the results of the fit with Eqs. (12) and (13). The fits typically differ from the experimental distribution by less than $5 \%$.

In comparison with Refs. [19,20], where the transverse profile exist only for distances less than $250 \mathrm{~mm}$, our more extended profiles (up to $650 \mathrm{~mm}$ ) require that the third exponential term be introduced. The parameters $a_{i}$ and $\lambda_{i}$, obtained by fitting, are listed in Table 1 . The values of the parameter $E_{0}$, the average energy shower deposition in a given depth segment, are listed in Table 4.

We have compared our values of $\lambda_{1}$ and $\lambda_{2}$ with the ones from the conventional iron-scintillator calorimeter described in Ref. [20]. At $100 \mathrm{GeV}$, our results for the entire calorimeter are $23 \pm 1$ and $58 \pm 4 \mathrm{~mm}$ for $\lambda_{1}$ and $\lambda_{2}$, respectively. They agree well with the ones from Ref. [20], which are $18 \pm 3$ and $57 \pm 4 \mathrm{~mm}$.

We determined the FWHM of energy deposition profiles (Figs. 4 and 5) using formula (14). The characteristic FWHM are found to be approximately equal to transverse tower size. The relative differences of FWHM from transverse tower size, $(\mathrm{FWHM}-h) / h$, amount to $2 \%$ for depth segment 1 , depth segment 2 and for the entire calorimeter, $7 \%$ for depth segment 3 and $15 \%$ for depth segment 4.

Fig. 6 shows the calculated marginal density function $f(z)$ and the energy deposition function, $E(z) / h$, at various transverse sizes of tower $h=50$, 200, 300 and $800 \mathrm{~mm}$ using the obtained parameters for the entire calorimeter. As a result of the 
Table 1

The parameters $a_{i}$ and $\lambda_{i}$ obtained by fitting the transverse shower profiles for four depth segments and the entire calorimeter at $100 \mathrm{GeV}$

\begin{tabular}{lllllll}
\hline Depth & $x\left(\lambda_{\pi}^{\mathrm{Fe}}\right)$ & $a_{1}$ & $\lambda_{1}(\mathrm{~mm})$ & $a_{2}$ & $\lambda_{2}(\mathrm{~mm})$ & $a_{3}$ \\
\hline 1 & 0.6 & $0.88 \pm 0.07$ & $17 \pm 2$ & $0.12 \pm 0.07$ & $48 \pm 14$ & $0.004 \pm 0.002$ \\
2 & 2.0 & $0.79 \pm 0.06$ & $25 \pm 2$ & $0.20 \pm 0.06$ & $52 \pm 6$ & $0.014 \pm 0.006$ \\
3 & 3.8 & $0.69 \pm 0.03$ & $32 \pm 8$ & $0.28 \pm 0.03$ & $71 \pm 13$ & $0.029 \pm 0.005$ \\
4 & 6.0 & $0.41 \pm 0.05$ & $51 \pm 10$ & $0.52 \pm 0.06$ & $73 \pm 18$ & $0.07 \pm 0.03$ \\
All four & & $0.78 \pm 0.08$ & $23 \pm 1$ & $0.20 \pm 0.08$ & $58 \pm 4$ & $0.015 \pm 0.004$ \\
\hline
\end{tabular}

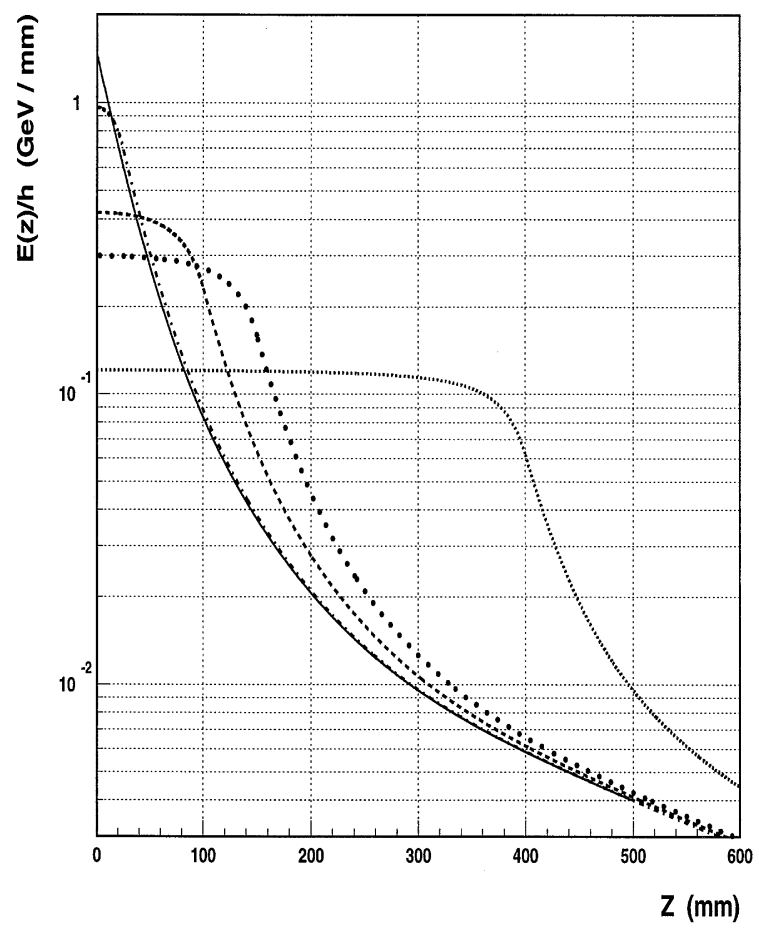

Fig. 6. The calculated marginal density function $f(z)$ (the solid line) and the energy deposition function, $E(z) / h$, for various transverse sizes of a tower $(h): 50 \mathrm{~mm}$ (the dash-dotted line), $200 \mathrm{~mm}$ (the dashed line), $300 \mathrm{~mm}$ (the thick dotted line), $800 \mathrm{~mm}$ (the thin dotted line). The parameters for the entire calorimeter (See Table 1) are used in the calculations.

volume integration, the $\operatorname{sharp} f(z)$ is transformed to the wide function $E(z) / h$, which clearly shows its relationship to the transverse width of a tower. The value of FWHM are $40 \mathrm{~mm}$ for $f(z)$ and $204 \mathrm{~mm}$ for $E(z) / h$ at $h=200 \mathrm{~mm}$. Note that the transverse dimensions of a tower vary from 300 to $800 \mathrm{~mm}$ for

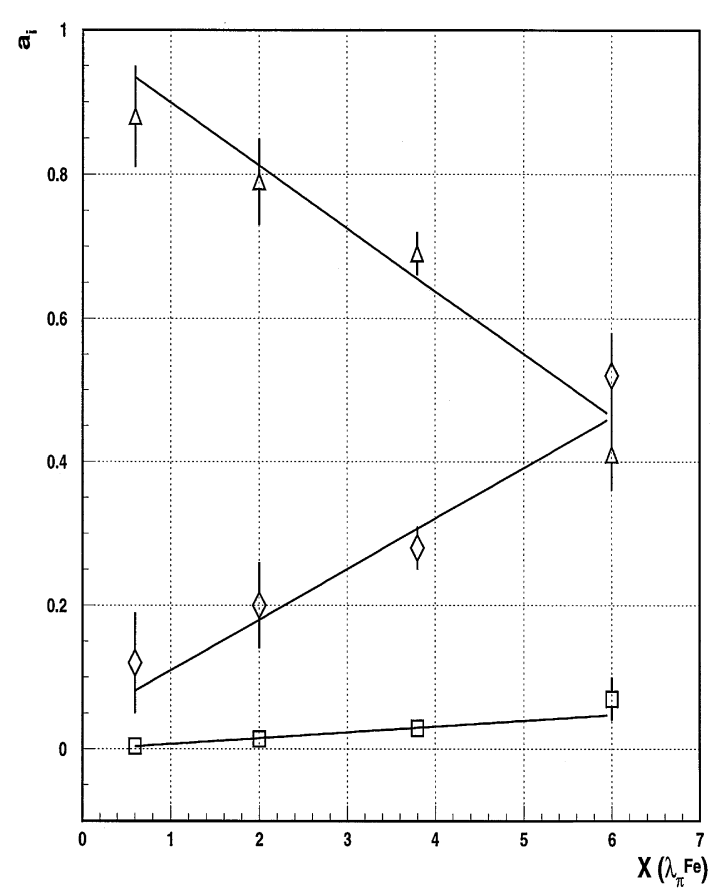

Fig. 7. $X$ dependences of the parameters $a_{i}$ : the triangles are the $a_{1}$ parameter, the diamonds are the $a_{2}$, the squares are the $a_{3}$.

the different depth segments in final ATLAS Tile Calorimeter design. The difference between $f(0)$ and $E(0) / h$ becomes less than $5 \%$ only at $h$ less than $6 \mathrm{~mm}$.

The parameters $a_{i}$ and $\lambda_{i}$ as a function $x$ (in units of $\lambda_{\pi}^{\mathrm{Fe}}$ ) are displayed in Figs. 7 and 8 . Here $\lambda_{\pi}^{\mathrm{Fe}}=207 \mathrm{~mm}$ is the nuclear interaction length for pions in iron [18]. In these calculations, the effect of the $10^{\circ}$ incidence beam angle has been corrected. As can be seen from Figs. 7 and 8, the value of 


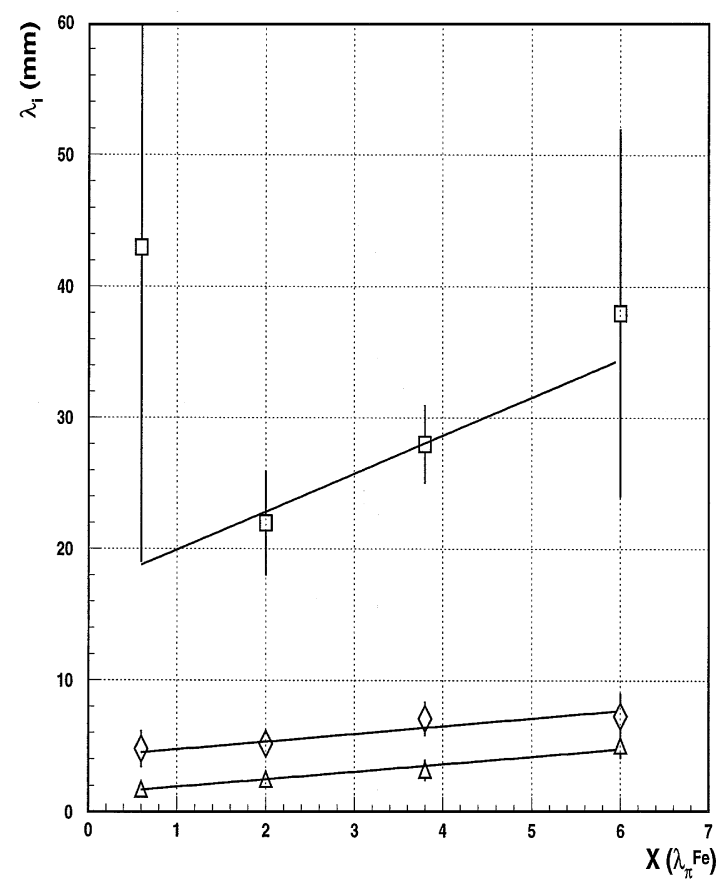

Fig. 8. $X$ dependences of the parameters $\lambda_{i}$ : the triangles are the $\lambda_{1}$ parameter, the diamonds are the $\lambda_{2}$, the squares are the $\lambda_{3}$.

$a_{1}$ decreases and the values of the remaining parameters, $a_{2}, a_{3}$ and $\lambda_{i}$ increase as the shower develops. This is a reflection of the fact that as the hadronic shower propagates into the calorimeter it becomes broader. Note also that the $a_{i}$ and $\lambda_{i}$ parameters demonstrate linear behaviour as a function of $x$. The lines shown are fits to the linear equations

$a_{i}(x)=\alpha_{i}+\beta_{i} x$

and

$\lambda_{i}(x)=\gamma_{i}+\delta_{i} x$.
The values of the parameters, $\alpha_{i}, \beta_{i}, \gamma_{i}$ and $\delta_{i}$ are presented in Table 2. It is interesting to note that the linear behaviour of the slope exponential was also observed for the low-density fine-grained flash chamber calorimeter [4]. However, some non-linear behaviour of the slope of the halo component was demonstrated for the uranium-scintillator ZEUS calorimeter at interaction lengths more than $5 \lambda_{I}[21]$.

Similar results were obtained for the cumulative function distributions. The cumulative function $F(x)$ is given by

$F(z)=\sum_{k=1}^{4} F^{k}(z)$

where $F^{k}(z)$ is the cumulative function for depth segment $k$. For each event, $F^{k}(z)$ is

$F^{k}(z)=\sum_{i=1}^{i_{\max }} \sum_{j=1}^{5} E_{i j k}$

where $i_{\max }=1, \ldots, 5$ is the last tower number in the sum.

Figs. 9 and 10 present the cumulative functions for four depth segments and for the entire calorimeter. The curves are fits of Eqs. (16) and (17) to the data. Systematic and statistical errors are again added in quadrature. The results of the cumulative function fits are less reliable and in what follows we use the results from energy depositions in a tower.

However, the marginal density functions determined by the two methods (by using the energy deposition spectrum and the cumulative function) are in reasonable agreement.

\section{Radial hadronic shower energy density}

Using formula (11) and the values of the parameters $a_{i}, \lambda_{i}$, given in Table 1 , we have determined

Table 2

The value of the parameters $\alpha_{i}, \beta_{i}, \lambda_{i}$ and $\delta_{i}$

\begin{tabular}{|c|c|c|c|c|c|}
\hline & $\alpha_{i}$ & $\beta_{i}\left(1 / \lambda_{\pi}\right)$ & & $\gamma_{i}(\mathrm{~mm})$ & $\delta_{i}\left(\mathrm{~mm} / \lambda_{\pi}\right)$ \\
\hline$a_{1}$ & $0.99 \pm 0.06$ & $-0.088 \pm 0.015$ & $\lambda_{1}$ & $13 \pm 2$ & $6 \pm 1$ \\
\hline$a_{2}$ & $0.04 \pm 0.06$ & $0.071 \pm 0.015$ & $\lambda_{2}$ & $42 \pm 10$ & $6 \pm 4$ \\
\hline$a_{3}$ & $-0.001 \pm 0.002$ & $0.008 \pm 0.002$ & $\lambda_{3}$ & $170 \pm 80$ & $29 \pm 23$ \\
\hline
\end{tabular}



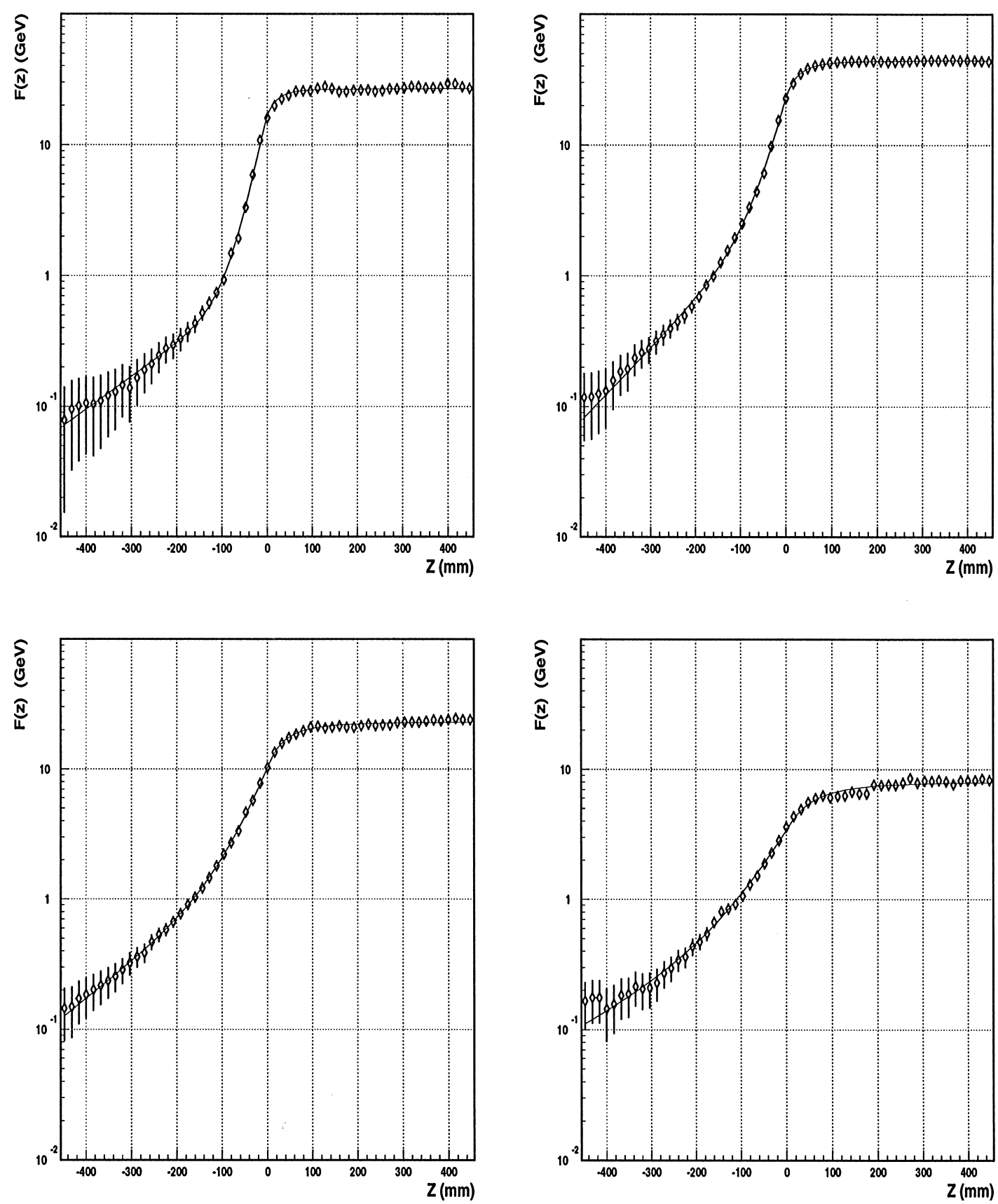

Fig. 9. Cumulative functions $F(z)$ for depth segments $1-4$ as a function of the $z$ coordinate: top left is for depth segment 1 , top right is for depth segment 2, bottom left is for depth segment 3, bottom right is for depth segment 4 . Statistical and systematic errors, summed in quadrature, are shown. Curves are fits of Eqs. (16) and (17) to the data. 




Fig. 10. The cumulative function $F(z)$ for the entire calorimeter as a function of the $z$ coordinate. Only statistical errors are shown. Curves are fits of Eqs. (16) and (17) to the data.

the underlying radial hadronic shower energy density functions, $\Phi(r)$. The results are shown in Fig. 11 for depth segments 1-4 and in Fig. 12 for the entire calorimeter. The contributions of the three terms of $\Phi(r)$ are also shown.

The functions $\Phi(r)$ for separate depth segments of a calorimeter are given in this paper for the first time. The function $\Phi(r)$ for the entire calorimeter was previously given for a lead-scintillating fibre calorimeter [14]; it is given here for the first time for an iron-scintillator calorimeter. The analytical functions giving the radial energy density for different depth segments allow to easily obtain the shower energy deposition in any calorimeter cell, shower containment fractions and the cylinder radii for any given shower containment fraction.

The function $\Phi(r)$ for the entire calorimeter has been compared with the one for the lead-scintillating fibre calorimeter of Ref. [14], that has about the same effective nuclear interaction length for pions (namely $251 \mathrm{~mm}$ for the tile and $244 \mathrm{~mm}$ for the fibre calorimeter [18]). The two radial density functions are rather similar as seen in Fig. 13. The lead-scintillating fibre calorimeter density function $\Phi(r)$, which was obtained from a $80 \mathrm{GeV} \pi^{-}$grid scan at an angle of $2^{\circ}$ with respect to the fibre direction, was parametrised using formula (6) with $b_{1}=0.169 \mathrm{pC} / \mathrm{mm}, b_{2}=0.677 \mathrm{pC} / \mathrm{mm}, \mu_{1}=$ $140 \mathrm{~mm}, \mu_{2}=42.4 \mathrm{~mm}$. For the sake of comparing the radial density functions of the two calorimeters, the distribution from Ref. [14] was normalised to the $\Phi(r)$ of the Tile Calorimeter. Precise agreement between these functions should not be expected because of the effect of the different absorber materials used in the two detectors (e.g. the radiation/interaction length ratio for the Tile Calorimeter is three times larger than for lead-scintillating fibre calorimeter [18]), the values of $e / h$ are different, as is hadronic activity of showers because fewer neutrons are produced in iron than in lead [22,23].

\section{Radial containment}

Another issue on which new results are presented here is the longitudinal development of shower transverse dimensions. The parametrisation of the radial density function, $\Phi(r)$, was integrated to yield the shower containment as a function of the radius, $I(r)$. Fig. 14 shows the transverse containment of the pion shower, $I(r)$, as a function of $r$ for four depth segments and for the entire calorimeter.

In Table 3 and Fig. 15 the radii of cylinders for the given shower containment $(90 \%, 95 \%$ and $99 \%$ ) extracted from Fig. 14 as a function of depth are shown. The centres of depth segments, $x$, are given in units of $\lambda_{\pi}^{\mathrm{Fe}}$. Solid lines are the linear fits to the data: $r(90 \%)=(85 \pm 6)+(37 \pm 3) x, r(95 \%)$ $=(134 \pm 9)+(45 \pm 3) x, r(99 \%)=(349 \pm 7)+$ $(22 \pm 2) x(\mathrm{~mm})$. As can be seen, these containment radii increase linearly with depth. Such a linear increase of $95 \%$ lateral shower containment with depth is also observed in an other iron-scintillator calorimeter at 50 and $140 \mathrm{GeV}$ [24]. It is interesting to note that the shower radius for $95 \%$ radial containment for the entire calorimeter is equal to $\lambda_{\pi}^{\text {eff }}=251 \mathrm{~mm}$ [18] which justifies the frequently encountered statement that $r(95 \%) \approx \lambda_{I} \quad[22]$, where $\lambda_{I}$ is $\lambda_{\pi}^{\text {eff }}$ in our case. For the entire Tile 

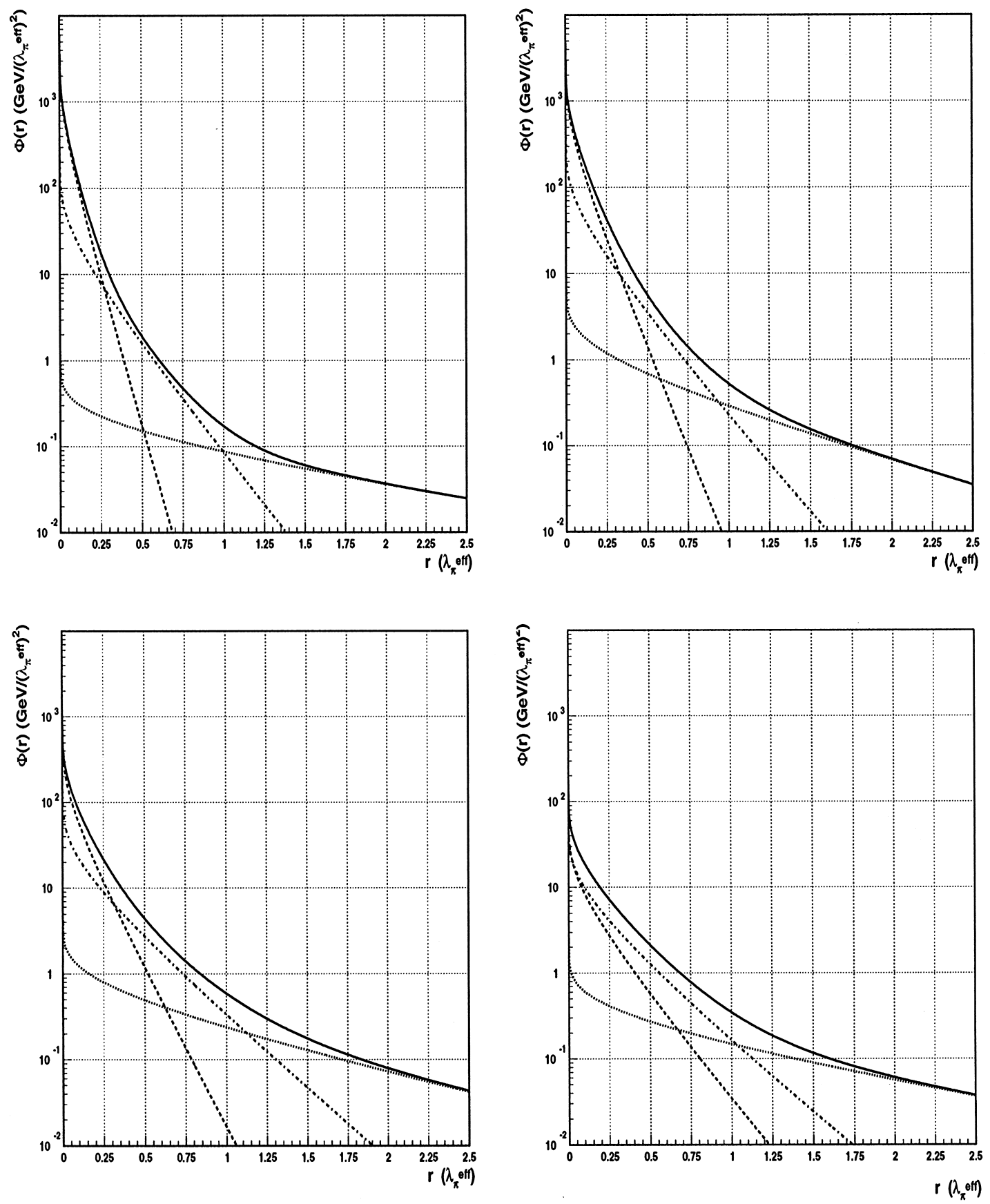

Fig. 11. Radial energy density, $\Phi(r)$, as a function of $r$ for Tile Calorimeter for depth segments 1-4: top left is for depth segment 1, top right is for depth segment 2, bottom left is for depth segment 3, bottom right is for depth segment 4 . The solid lines are the energy densities $\Phi(r)$, the dashed lines are the contribution from the first term from Eq. (11), the dash-dotted lines are the contribution from the second term, the dotted lines are the contribution from the third term. 


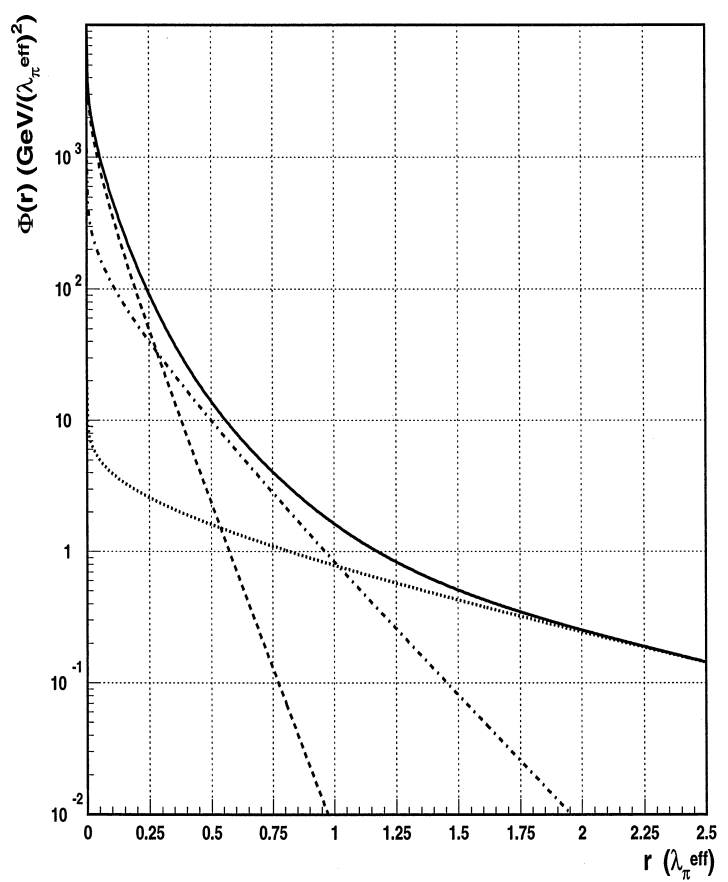

Fig. 12. The radial energy density as a function of $r$ (in units of $\lambda_{\pi}^{\text {eff }}$ ) for Tile Calorimeter (the solid line), the contribution to $\Phi(r)$ from the first term in equation (11) (the dashed line), the contribution to $\Phi(r)$ from the second term (the dash-dotted line), the contribution to $\Phi(r)$ from the third term (the dotted line).

Calorimeter the $99 \%$ containment radius is equal to $1.7 \pm 0.1 \lambda_{\pi}^{\text {eff }}$.

Based on our study, we believe that it is a poor approximation to regard the values obtained from the marginal density function or the energy depositions in strips as the measure of the transverse shower containment, as was done in Ref. [4]. In that paper the value of $1.1 \lambda_{\pi}^{\text {eff }}$ was obtained for $99 \%$ containment at $100 \mathrm{GeV}$, and the conclusion was drawn that their "result is consistent with the rule of thumb that a shower is contained within a cylinder of radius equal to the interaction length of a calorimeter material". However Tile Calorimeter measurements show that the cylinder radius for $99 \%$ shower containment is about two interaction lenghts. If we extract the lateral shower containment dimension using instead the integrated function $F(z)$, given in Fig. 10, we obtain the value of $300 \mathrm{~mm}$ or $1.2 \lambda_{\pi}^{\text {eff }}$, which agrees with Ref. [4].

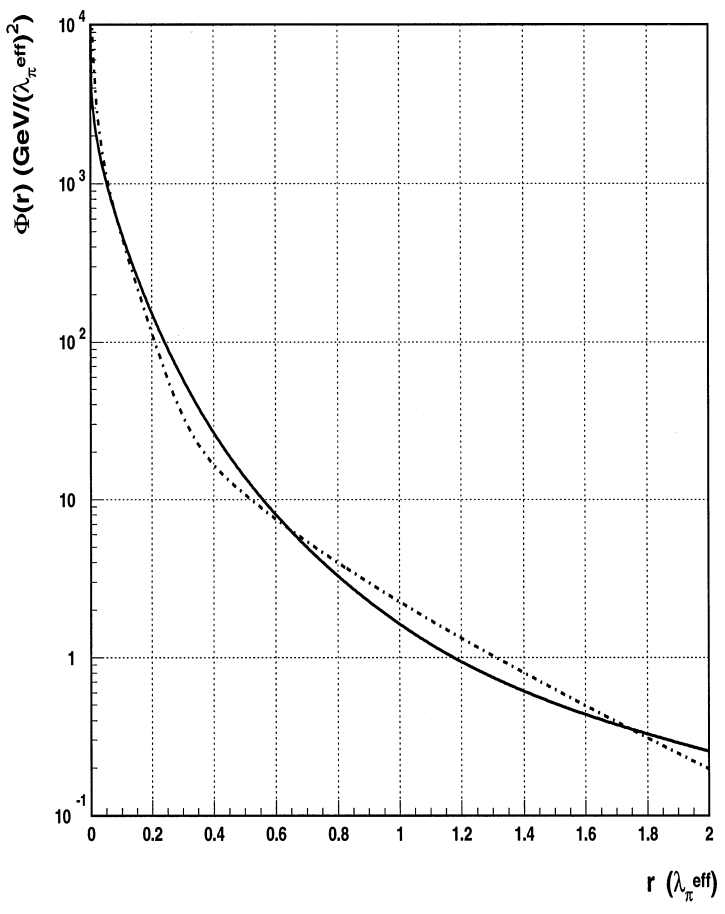

Fig. 13. Comparison of the radial energy densities as a function of $r$ (in units of $\lambda_{\pi}^{\text {eff }}$ ) for Tile Calorimeter (the solid line) and lead-scintillating fibre calorimeter (the dash-dotted line).

\section{Longitudinal profile}

We have examined the differential deposition of energy $\Delta E / \Delta x$ as a function of $x$, the distance along the shower axis. Table 4 lists the centres in $x$ of the depth segments, $x$, and the lenghts along $x$ of the depth segments, $\Delta x$, in units of $\lambda_{\pi}^{\mathrm{Fe}}$, the average shower energy depositions in various depth segments, $E_{0}$, and the energy depositions per interaction length $\lambda_{\pi}^{\mathrm{Fe}}, \Delta E / \Delta x$. Note that the values of $E_{0}$ have been obtained taking into account the longitudinal energy leakage which amounts to $1.8 \mathrm{GeV}$ for $100 \mathrm{GeV}$ [12].

Our values of $\Delta E / \Delta x$ together with the data of Ref. [25] and Monte Carlo predictions (GEANTFLUKA + MICAP) [26] are shown in Fig. 16. The longitudinal energy deposition for our calorimeter using longitudinal orientation of the scintillating tiles is in good agreement with that of a conventional iron-scintillator calorimeter. 


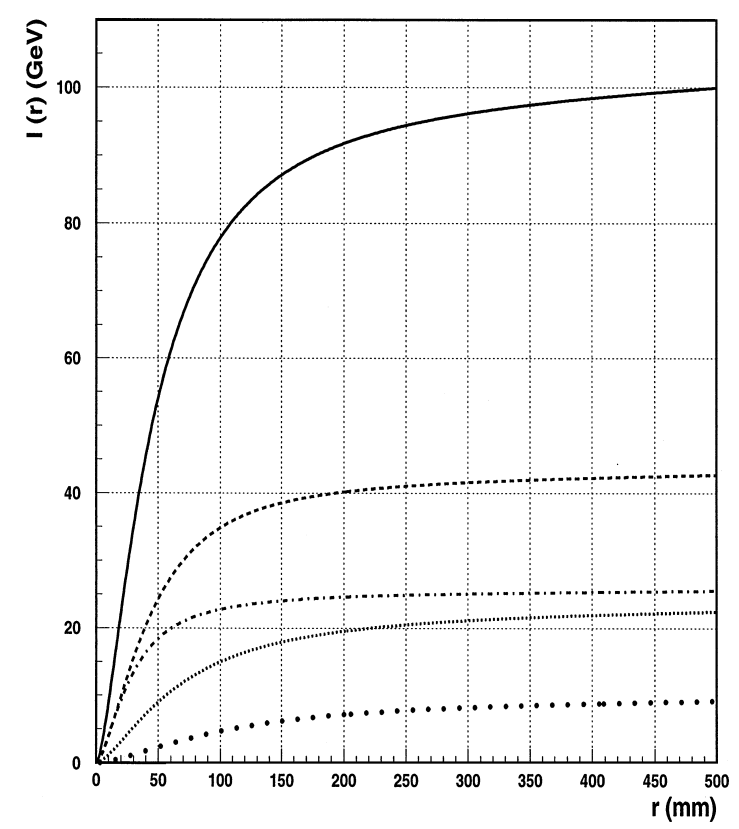

Fig. 14. Containment of shower $I(r)$ (the solid line) as a function of radius for the entire Tile Calorimeter. The dash-dotted line is the contribution from the first depth segment, the dashed line is the contribution from the second depth segment, the thin dotted line is the contribution from the third depth segment, the thick dotted line is the contribution from the fourth depth segment.

Table 3

The radii of cylinders for the given shower containment at $100 \mathrm{GeV}$

\begin{tabular}{llll}
\hline$x\left(\lambda_{\pi}^{\mathrm{Fe}}\right)$ & $\begin{array}{l}r\left(\lambda_{\pi}^{\text {eff }}\right) \\
90 \%\end{array}$ & $95 \%$ & $99 \%$ \\
\hline 0.6 & 0.44 & 0.64 & 1.43 \\
2.0 & 0.60 & 0.88 & 1.55 \\
3.8 & 0.92 & 1.27 & 1.75 \\
6.0 & 1.24 & 1.55 & 1.87 \\
All four & 0.72 & 1.04 & 1.67 \\
\hline
\end{tabular}

The longitudinal profile, $\Delta E / \Delta x$, may be approximated using two parametrisations. The first form is

$$
\frac{\mathrm{d} E(x)}{\mathrm{d} x}=\frac{E_{f} \beta^{\alpha+1}}{\Gamma(\alpha+1)} x^{\alpha} \mathrm{e}^{-\beta x}
$$

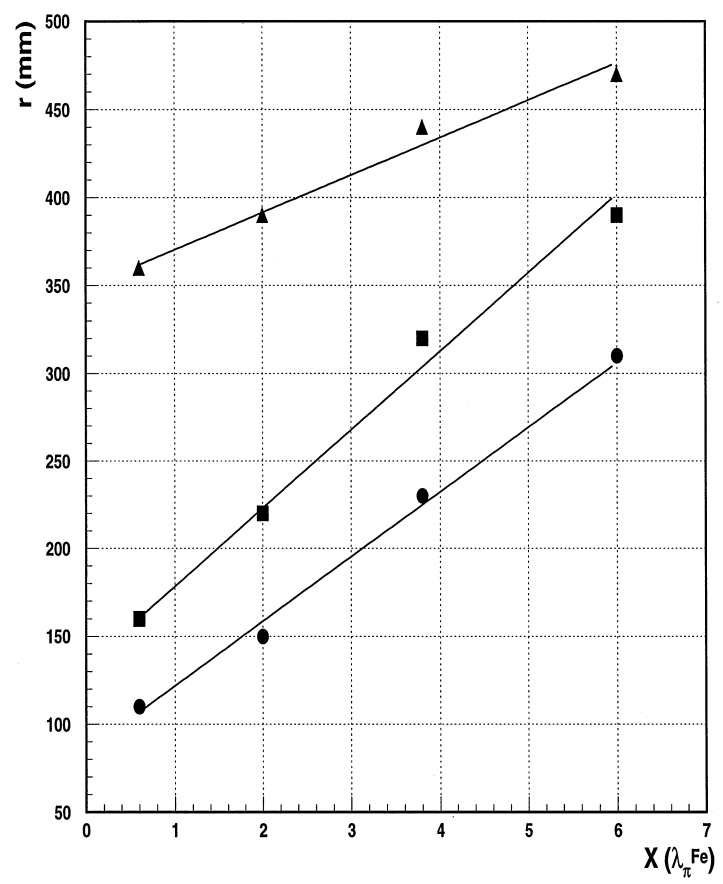

Fig. 15. The radii of cylinders for the given shower containment as a function of depths: the black circles are $90 \%$ of containment, the black squares are $95 \%$, the black triangles are $99 \%$.

where $E_{f}=E_{\mathrm{beam}}$, and $\alpha$ and $\beta$ are free parameters. Our data at $100 \mathrm{GeV}$ and those of Ref. [25] at $100 \mathrm{GeV}$ were jointly fit to this expression; the fit is shown in Fig. 16.

The second form is the analytical representation of the longitudinal shower profile from the front of the calorimeter,

$$
\begin{aligned}
\frac{\mathrm{d} E(x)}{\mathrm{d} x}= & N\left\{\frac{w X_{0}}{a}\left(\frac{x}{X_{0}}\right)^{a} \mathrm{e}^{-b x / X_{0}}\right. \\
& \times{ }_{1} F_{1}\left(1, a+1,\left(b-\frac{X_{0}}{\lambda_{I}}\right) \frac{x}{X_{0}}\right) \\
& +\frac{(1-w) \lambda_{I}}{a}\left(\frac{x}{\lambda_{I}}\right)^{a} \mathrm{e}^{-d x / \lambda_{I}} \\
& \left.\times{ }_{1} F_{1}\left(1, a+1,(d-1) \frac{x}{\lambda_{I}}\right)\right\}
\end{aligned}
$$

where ${ }_{1} F_{1}$ is the confluent hypergeometric function [27]. Here the depth variable, $x$, is the depth in 
Table 4

Average energy shower depositions at various depth segments at $100 \mathrm{GeV}$

\begin{tabular}{llrl}
\hline$x\left(\lambda_{\pi}^{\mathrm{Fe}}\right)$ & $\Delta x\left(\lambda_{\pi}^{\mathrm{Fe}}\right)$ & \multicolumn{1}{c}{$E_{0}(\mathrm{GeV})$} & $\Delta E / \Delta x\left(\mathrm{GeV} / \lambda_{\pi}^{\mathrm{Fe}}\right)$ \\
\hline 0.6 & 1.2 & $25.0 \pm 0.3$ & $20.8 \pm 0.3$ \\
2.0 & 1.6 & $42.8 \pm 0.2$ & $26.8 \pm 0.1$ \\
3.8 & 2.0 & $22.0 \pm 0.1$ & $11.0 \pm 0.1$ \\
6.0 & 2.4 & $8.4 \pm 0.5$ & $3.4 \pm 0.2$ \\
\hline
\end{tabular}

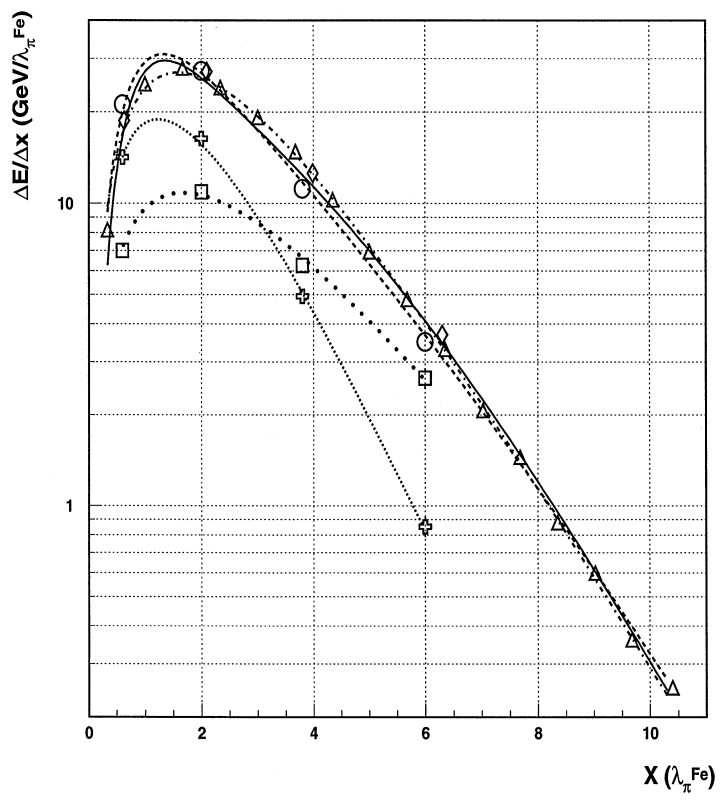

Fig. 16. The longitudinal profile (circles) of the hadronic shower at $100 \mathrm{GeV}$ as a function of the longitudinal coordinate $x$ in units of $\lambda_{\pi}^{\mathrm{Fe}}$. Open triangles are data from the calorimeter of Ref. [25], diamonds are the Monte Carlo (GEANT-FLUKA) predictions. The dash-dotted line is the fit by function (23), the solid line is calcuated with function (24) with parameters from Ref. [25], the dashed line is calculated with function (24) with parameters from Ref. [5]. The electromagnetic and hadronic components of the shower (crosses and squares), together with their fits using Eq. (23), are discussed in Section 9.

equivalent $\mathrm{Fe}, X_{0}$ is the radiation length in $\mathrm{Fe}$ and in this case $\lambda_{I}$ is $\lambda_{\pi}^{\mathrm{Fe}}$. The normalisation factor, $N$, is given by

$$
\begin{aligned}
N & =E_{\text {beam }} / \int_{0}^{\infty} \frac{\mathrm{d} E(x)}{\mathrm{d} x} \mathrm{~d} x \\
& =\frac{E_{\text {beam }}}{\lambda_{I} \Gamma(a)\left(w X_{0} b^{-a}+(1-w) \lambda_{I} d^{-a}\right)} .
\end{aligned}
$$

This form was suggested in Ref. [28] and derived by integration over the shower vertex positions of the longitudinal shower development from the shower origin

$\frac{\mathrm{d} E(x)}{\mathrm{d} x}=\int_{0}^{x} \frac{\mathrm{d} E_{\mathrm{s}}\left(x-x_{\mathrm{v}}\right)}{\mathrm{d} x} \mathrm{e}^{-x_{\mathrm{v}} / \lambda_{I}} \mathrm{~d} x_{\mathrm{v}}$

where $x_{\mathrm{v}}$ is the coordinate of the shower vertex. (This is necessary because with the Tile Calorimeter longitudinal segmentation the shower is not measured.) For the parametrisation of longitudinal shower development, the well-known parametrisation suggested by Bock et al. [5] has been used

$$
\begin{aligned}
\frac{\mathrm{d} E_{\mathrm{s}}(x)}{\mathrm{d} x}= & N\left\{w\left(\frac{x}{X_{0}}\right)^{a-1} \mathrm{e}^{-b x / X_{0}}\right. \\
& \left.+(1-w)\left(\frac{x}{\lambda_{I}}\right)^{a-1} \mathrm{e}^{-d x / \lambda_{I}}\right\},
\end{aligned}
$$

where $a, b, d, w$ are parameters.

We compare the form (24) to the experimental points at $100 \mathrm{GeV}$ using the parameters calculated in Refs. $[5,25]$. Note that now we are not performing a fit but checking how well the general form (24) together with two sets of parameters for iron-scintillator calorimeters describe our data. As shown in Fig. 16, both sets of parameters work rather well in describing the $100 \mathrm{GeV}$ data.

Turning next to the longitudinal shower development at different energies, in Fig. 17 our values of $\Delta E / \Delta x$ for $20-300 \mathrm{GeV}$ together with the data from Ref. [25] are shown. The solid and dashed lines are calculations with function (24) using parameters from Refs. [25,5], respectively. Again, we observe reasonable agreement between our data and the corresponding data for conventional iron-scintillator calorimeter on the one hand, and between data and the parametrisations described above. Note that the fit in Ref. [25] has been performed in the energy range from 10 to $140 \mathrm{GeV}$; hence the curves for 200 and $300 \mathrm{GeV}$ should be considered as extrapolations. It is not too surprising that at these energies the agreement is significantly worse, particularly at $300 \mathrm{GeV}$. In contrast, the parameters of Ref. [5] were derived from data spanning the range $15-400 \mathrm{GeV}$, and are in much closer agreement with our data. 


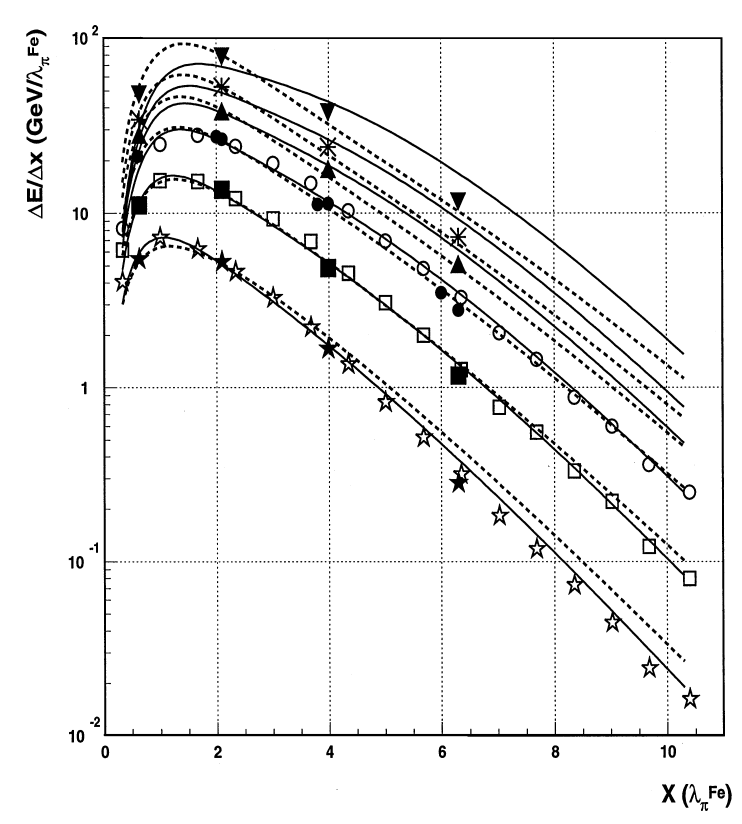

Fig. 17. Longitudinal profiles of the hadronic showers from $20 \mathrm{GeV}$ (open stars), $50 \mathrm{GeV}$ (open squares) and $100 \mathrm{GeV}$ (open circles) pions as a function of the longitudinal coordinate $x$ in units of $\lambda_{I}$ for a conventional iron-scintillator calorimeter [25] and of $20 \mathrm{GeV}$ (black stars), $50 \mathrm{GeV}$ (black squares), $100 \mathrm{GeV}$ (black circles), $150 \mathrm{GeV}$ (black up triangles), $200 \mathrm{GeV}$ (asterisks), $300 \mathrm{GeV}$ (black down triangles) for pions at $20^{\circ}$ and of $100 \mathrm{GeV}$ (black circles) for pions at $10^{\circ}$ for Tile iron-scintillator calorimeter. The solid lines are calculated with function (24) with parameters from Ref. [25]. The dashed lines are calculated with function (24) with parameters from Ref. [5].

\section{The parametrisation of hadronic showers}

The three-dimensional parametrisation for spatial hadronic shower development is

$\Psi(x, r)=\frac{\mathrm{d} E(x)}{\mathrm{d} x} \frac{\sum_{i=1}^{3}\left(a_{i}(x) / \lambda_{i}(x)\right) K_{0}\left(r / \lambda_{i}(x)\right)}{2 \pi \sum_{i=1}^{3} a_{i}(x) \lambda_{i}(x)}$

where $\mathrm{d} E(x) / \mathrm{d} x$, defined by Eq. (24), is the longitudinal energy deposition, the functions $a_{i}(x)$ and $\lambda_{i}(x)$ are given by Eqs. (19) and (20), and $K_{0}$ is the modified Bessel function.

This explicit three-dimensional parametrisation can be used as a convenient tool for many calorimetry problems requiring the integration of a shower energy deposition in a volume and the reconstruction of the shower coordinates.

\section{Electromagnetic fraction of hadronic showers}

One of the important issues in the understanding of hadronic showers is the electromagnetic component of the shower, i.e. the fraction of energy going into $\pi^{0}$ production and its dependence on radial and longitudinal coordinates, $f_{\pi^{0}}(r, x)$. Following Ref. [14], we assume that the electromagnetic part of a hadronic shower is the prominent central core, which in our case is the first term in expression (11) for the radial energy density function, $\Phi(r)$. Integrating $f_{\pi^{0}}$ over $r$ we get

$f_{\pi^{0}}=\frac{a_{1} \lambda_{1}}{\sum_{i=1}^{3} a_{i} \lambda_{i}}$.

For the entire Tile Calorimeter this value is $(53 \pm 3) \%$ at $100 \mathrm{GeV}$.

The observed $\pi^{o}$ fraction, $f_{\pi^{0}}$, is related to the intrinsic actual fraction, $f_{\pi^{0}}^{\prime}$ by the equation

$f_{\pi^{0}}(E)=\frac{e E_{\mathrm{em}}^{\prime}}{e E_{\mathrm{em}}^{\prime}+h E_{\mathrm{h}}^{\prime}}=\frac{e / h f_{\pi^{0}}^{\prime}(E)}{(e / h-1) f_{\pi^{0}}(E)+1}$

where $E_{\mathrm{em}}^{\prime}$ and $E_{\mathrm{h}}^{\prime}$ are the intrinsic electromagnetic and hadronic parts of shower energy, $e$ and $h$ are the coefficients of conversion of intrinsic electromagnetic and hadronic energies into observable signals, $f_{\pi^{0}}^{\prime}=E_{\mathrm{em}}^{\prime} /\left(E_{\mathrm{em}}^{\prime}+E_{\mathrm{h}}^{\prime}\right)$.

There are two analytic forms for the intrinsic $\pi^{0}$ fraction suggested by Groom [29]

$f_{\pi^{0}}^{\prime}(E)=1-\left(\frac{E}{E_{0}^{\prime}}\right)^{m-1}$

and Wigmans [30]

$f_{\pi^{0}}^{\prime}(E)=k \ln \left(\frac{E}{E_{0}^{\prime}}\right)$

where $E_{0}^{\prime}=1 \mathrm{GeV}, m=0.85$ and $k=0.11$. We calculated $f_{\pi^{\circ}}$ using the value $e / h=1.34 \pm 0.03$ for our calorimeter $[2,31]$ and obtained the curves shown in Fig. 18.

Our result at $100 \mathrm{GeV}$ is compared in Fig. 18 to the modified Groom and Wigmans parametrisations and to results from the Monte Carlo codes CALOR [23], GEANT-GEISHA [26] and GEANT-CALOR [32] (the latter code is an implementation of CALOR89 differing from 


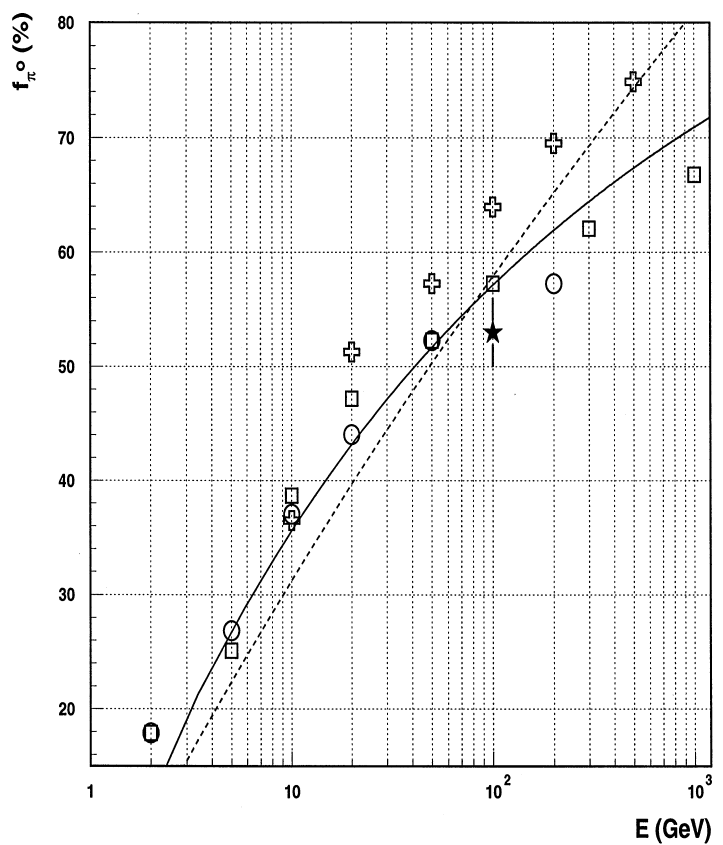

Fig. 18. The fraction $f_{\pi^{\circ}}$ in hadronic showers versus the beam energy. The star is our data, the solid curve is the Groom parametrisation, the dashed curve is the Wigmans parametrisation, squares are the GEANT-CALOR predictions, circles are the GEANT-GHEISHA predictions and crosses are the CALOR predictions.

GEANT-FLUKA only for hadronic interactions below $10 \mathrm{GeV}$ ). Note that the Monte Carlo calculations were performed for the intrinsic $\pi^{0}$ fraction, $f_{\pi^{0}}^{\prime}(E)$, and therefore the results were modified by us according to Eq. (30). As can be seen from Fig. 18, our calculated value of $f_{\pi^{\circ}}$ is about one standard deviation lower than two of the Monte Carlo results and the Groom and Wigmans parametrisations.

Fig. 19 shows the fractions $f_{\pi^{\circ}}(r)$ as a fraction of $r$. As can be seen, the fractions $f_{\pi^{\circ}}(r)$ for the entire calorimeter and for depth segments 1-3 amount to about $90 \%$ as $r \rightarrow 0$ and decrease to about $1 \%$ as $r \rightarrow \lambda_{\pi}^{\text {eff }}$. However for depth segment 4 the value of $f_{\pi^{0}}(r)$ amounts to only $50 \%$ as $r \rightarrow 0$ and decreases slowly to about $10 \%$ as $r \rightarrow \lambda_{\pi}^{\text {eff }}$.

Fig. 20 shows the values of $f_{\pi^{0}}(x)$ as a function of $x$, as well as the linear fit which gives $f_{\pi^{0}}(x)=(75 \pm 2)-(8.4 \pm 0.4) x(\%)$.

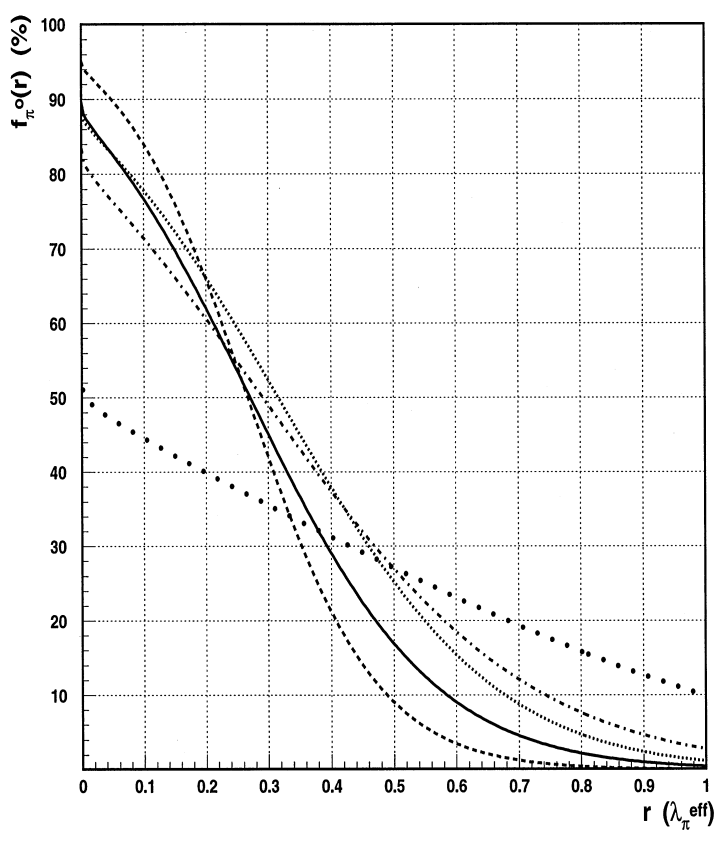

Fig. 19. The fraction $f_{\pi^{0}}$ of hadronic showers as a function of radius. The solid line is the $f_{\pi^{\circ}}(r)$ for the entire Tile Calorimeter. The dash-dotted line is the $f_{\pi^{\circ}}(r)$ for the first depth segment, the dashed line is the $f_{\pi^{0}}(r)$ for the second depth segment, the thin dotted line is the $f_{\pi^{0}}(r)$ for the third depth segment, the thick dotted line is the $f_{\pi^{0}}(r)$ for the fourth depth segment.

Using the values of $f_{\pi^{0}}(x)$ and energy depositions for various depth segments, we obtained the contributions from the electromagnetic and hadronic parts of hadronic showers in Fig. 16. The curves represent a fit to the electromagnetic and hadronic components of the shower using Eq. (23). $E_{\mathrm{f}}$ is set equal to $f_{\pi^{0}} E_{\text {beam }}$ for the electromagnetic fraction and $\left(1-f_{\pi^{0}}\right) E_{\text {beam }}$ for the hadronic fraction. The electromagnetic component of a hadronic shower rise and decrease more rapidly than the hadronic one $\left(\alpha_{\mathrm{em}}=1.4 \pm 0.1, \alpha_{\mathrm{h}}=1.1 \pm 0.1, \beta_{\mathrm{em}}=1.12 \pm\right.$ $\left.0.04, \beta_{\mathrm{h}}=0.65 \pm 0.05\right)$. The shower maximum position $\left(x_{\max }=(\alpha / \beta) \lambda_{\pi}^{\mathrm{Fe}}\right)$ occurs at a shorter distance from the calorimeter front face $\left(x_{\max }^{\mathrm{em}}=1.23 \lambda_{\pi}^{\mathrm{Fe}}, x_{\max }^{\mathrm{h}}=1.85 \lambda_{\pi}^{\mathrm{Fe}}\right)$. At depth segments greater than $4 \lambda_{\pi}^{\mathrm{Fe}}$, the hadronic fraction of the shower begins to dominate. This is natural since the energy of the secondary hadrons is too low to permit significant pion production. 


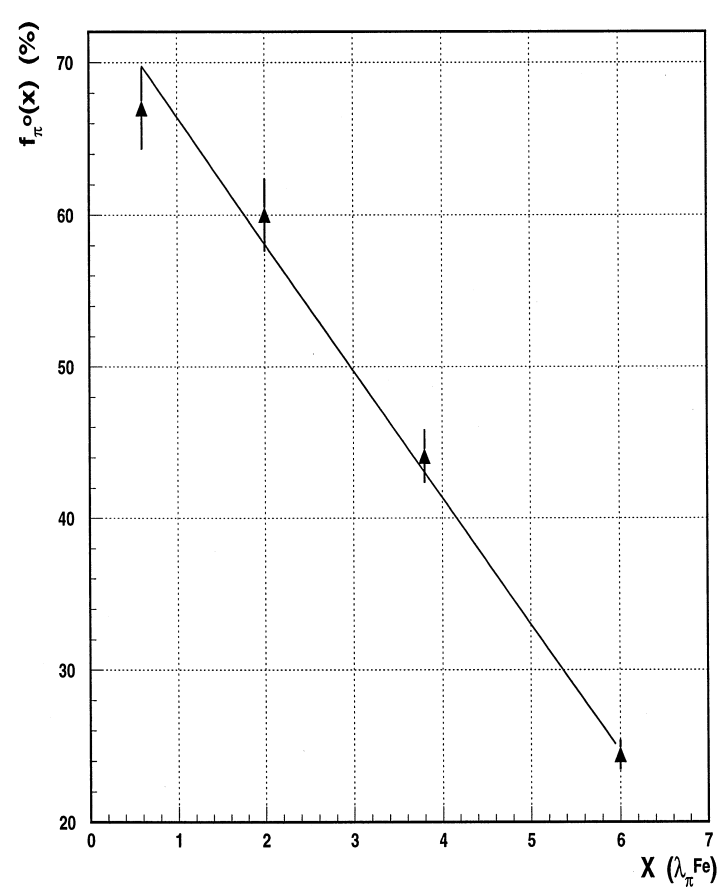

Fig. 20. The $f_{\pi^{\circ}}(x)$ fractions of hadronic showers as a function of $x$.

\section{Summary and conclusions}

We have investigated the lateral development of hadronic showers using $100 \mathrm{GeV}$ pion beam data at an incidence angle of $\Theta=10^{\circ}$ for impact points $z$ in the range from -360 to $200 \mathrm{~mm}$ and the longitudinal development of hadronic showers using 20-300 $\mathrm{GeV}$ pion beams at an incidence angle of $\Theta=20^{\circ}$.

Some useful formulae for the investigation of lateral profiles have been derived using a threeexponential form of the marginal density function $f(z)$.

We have obtained for four depth segments and for the entire calorimeter: energy depositions in towers, $E(z)$; cumulative functions, $F(z)$; underlying radial energy densities, $\Phi(r)$; the contained fraction of a shower as a function of radius, $I(r)$; the radii of cylinders for a given shower containment fraction; the fractions of the electromagnetic and hadronic parts of a shower; the differential longitudinal energy deposition $\Delta E / \Delta x$; and a three-dimensional hadronic shower parametrisation.
We have compared our data with those from a conventional iron-scintillator calorimeter, those from a lead-scintillator fibre calorimeter, and with Monte Carlo calculations. We have found that there is general reasonable agreement in the behaviour of the Tile Calorimeter radial density functions and those of the lead-scintillating fibre-calorimeter; that the longitudinal profile agrees with that of a conventional iron-scintillator calorimeter and with the Monte Carlo predictions; that the value at $100 \mathrm{GeV}$ of the calculated fraction of energy going into $\pi^{0}$ production in a hadronic shower, $f_{\pi^{0}}$, agrees with the Groom and Wigmans parametrisations and with some of the Monte Carlo predictions.

The three-dimensional parametrisation of hadronic showers that we obtained allows direct use in any application that requires volume integration of shower energy depositions and position reconstruction. The experimental data on the transverse and longitudinal profiles, the radial energy densities and the three-dimensional hadronic shower parametrisation are useful for understanding the performance of the Tile Calorimeter, but might find broader application in Monte Carlo modelling of hadronic showers, in particular in fast simulations, and for future calorimeters design.

\section{Acknowledgements}

This paper is the result of the efforts of many people from the ATLAS Collaboration. The authors are greatly indebted to the entire Collaboration for their test beam setup and data taking. We are grateful to the staff of the SPS, and in particular to Konrad Elsener, for the excellent beam conditions and assistance provided during our tests.

\section{References}

[1] ATLAS Collaboration, ATLAS Technical Proposal for a General-Purpose pp Experiment at the Large Hadron Collider, CERN/LHCC/94-93, CERN, Geneva, Switzerland, 1994.

[2] ATLAS Collaboration, ATLAS Tile Calorimeter Technical Design Report, ATLAS TDR 3, CERN/LHCC/9642, CERN, Geneva, Switzerland, 1996. 
[3] R.K. Bock, A. Vasilescu, The Particle Detector Briefbook, Springer, Berlin, 1998.

[4] W.J. Womersley et al., Nucl. Instr. and Meth. A 267 (1988) 49.

[5] R.K. Bock et al., Nucl. Instr. and Meth. 186 (1981) 533.

[6] G. Grindhammer, Nucl. Instr. and Meth. A 289 (1990) 469.

[7] R. Brun et al., Proceedings of the II International Conference on Cal. in HEP, Capri, Italy, 1991, p. 82.

[8] E. Berger et al., CERN/LHCC 95-44, CERN, Geneva, Switzerland, 1995.

[9] M. Lokajicek et al., ATLAS Internal Note, TILECALNo-64, CERN, Geneva, Switzerland, 1995.

[10] F. Ariztizabal et al., Nucl. Instr. and Meth. A 349 (1994) 384.

[11] M. Lokajicek et al., ATLAS Internal Note, TILECALNo-63, CERN, Geneva, Switzerland, 1995.

[12] J.A. Budagov, Y.A. Kulchitsky, V.B. Vinogradov et al., JINR, El-96-180, Dubna, Russia, 1996; ATLAS Internal note, TILECAL-No-75, CERN, Geneva, Switzerland, 1996.

[13] R.M. Barnett et al., Rev Particle Physics, Probability, Phys. Rev. D 54 (1996).

[14] D. Acosta et al., Nucl. Instr. and Meth. A 316 (1992) 184.

[15] A.A. Lednev et al., Nucl. Instr. and Meth. A 366 (1995) 292.

[16] G.A. Akopdijanov et al., Nucl. Instr. and Meth. 140 (1977) 441.

[17] E.T. Whitteker, G.N. Watson, A Course of Modern Analysis, Cambridge, Univ. Press, Cambridge, 1927.

[18] J.A. Budagov, Y.A. Kulchitsky, V.B. Vinogradov et al., JINR, E1-97-318, Dubna, Russia, 1997; ATLAS Internal note, TILECAL-No-127, CERN, Geneva, Switzerland, 1997.
[19] O.P. Gavrishchuk et al., JINR, P1-91-554, Dubna, Russia, 1991.

[20] F. Binon et al., Nucl. Instr. and Meth. A 206 (1983) 373.

[21] F. Barreiro et al., Nucl. Instr. and Meth. A 292 (1990) 259.

[22] C.W. Fabjan, T. Ludlam, Ann. Rev. Nucl. Part. Sci. 32 (1982).

[23] T.A. Gabriel et al., Nucl. Instr. and Meth. A 338 (1994) 336.

[24] M. Holder et al., Nucl. Instr. and Meth. 151 (1978) 69.

[25] E. Hughes, Proceedings of the I International Conference on Calor. in HEP, p. 525, FNAL, Batavia, 1990.

[26] A. Juste, ATLAS Internal note, TILECAL-No.69, CERN, Geneva, Switzerland, 1995.

[27] M. Abramovitz, I.A. Stegun (Eds.), Handbook of Mathematical Functions, National Bureau of Standards, Applied Mathematics, Columbia Univ. Press, New York, 1964.

[28] Y.A. Kulchitsky, V.B. Vinogradov, Nucl. Instr. and Meth. A 413 (1998) 484.

[29] D. Groom, Proceedings of the Workshop on Calorimetry for the Supercollider, Tuscaloosa, Alabama, USA, 1990.

[30] R. Wigmans, Nucl. Instr. and Meth. A 265 (1988) 273.

[31] J.A. Budagov, Y.A. Kulchitsky, V.B. Vinogradov et al., JINR, E1-95-513, Dubna, Russia, 1995; ATLAS Internal note, TILECAL-No-27, CERN, Geneva, Switzerland, 1995.

[32] M. Bosman, Establishing requirements from the point of view of the ATLAS hadronic barrel calorimeter, ATLAS Workshop of Shower Models, 15-16 September 1997, CERN, Geneva, Switzerland, 1997. 